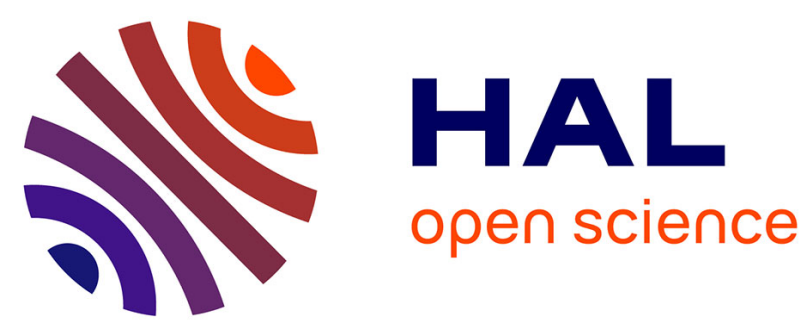

\title{
Evolutionary clustering for categorical data using parametric links among multinomial mixture models
}

Md Abul Hasnat, Julien Velcin, Stephane Bonnevay, Julien Jacques

\section{To cite this version:}

Md Abul Hasnat, Julien Velcin, Stephane Bonnevay, Julien Jacques. Evolutionary clustering for categorical data using parametric links among multinomial mixture models. Econometrics and Statistics , 2017, 3, pp.141-159. 10.1016/j.ecosta.2017.03.004 . hal-01204613v3

\section{HAL Id: hal-01204613 \\ https://hal.inria.fr/hal-01204613v3}

Submitted on 27 Feb 2019

HAL is a multi-disciplinary open access archive for the deposit and dissemination of scientific research documents, whether they are published or not. The documents may come from teaching and research institutions in France or abroad, or from public or private research centers.
L'archive ouverte pluridisciplinaire HAL, est destinée au dépôt et à la diffusion de documents scientifiques de niveau recherche, publiés ou non, émanant des établissements d'enseignement et de recherche français ou étrangers, des laboratoires publics ou privés. 


\title{
Evolutionary clustering for categorical data using parametric links among multinomial mixture models
}

\author{
Md. Abul Hasnat ${ }^{\mathrm{a}}$, Julien Velcin ${ }^{\mathrm{a}}$, Stephane Bonnevay ${ }^{\mathrm{b}}$, Julien Jacques ${ }^{\mathrm{a}, *}$ \\ ${ }^{a}$ Université de Lyon, Université Lyon 2 \& ERIC \\ ${ }^{b}$ Université de Lyon, Université Lyon 1 \& ERIC
}

\begin{abstract}
A novel evolutionary clustering method for temporal categorical data based on parametric links among the Multinomial mixture models is proposed. Besides clustering, the main goal is to interpret the evolution of clusters over time. To this aim, first the formulation of a generalized model that establishes parametric links among two Multinomial mixture models is proposed. Afterward, different parametric sub-models are defined in order to model the typical evolution of the clustering structure. Model selection criteria allow to select the best sub-model and thus to guess the clustering evolution. For the experiments, the proposed method is first evaluated with synthetic temporal data. Next, it is applied to analyze the annotated social media data. Results show that the proposed method is better than the state-of-the-art based on the common evaluation metrics. Additionally, it can provide interpretation about the temporal evolution of the clusters.
\end{abstract}

Keywords: evolutionary clustering, multinomial distribution, mixture model, model-based clustering, Twitter data

\section{Introduction}

In the recent years, the social media plays a significant role in many aspects of our daily activity. There exist numerous popular social media, such as Twitter or Facebook, where the users often provide their opinions about particular entity, e.g., persons (politician, actor), products consumed in the daily life, etc. A common method to analyze such data is - first use a clustering method to group the users/opinions, and then investigate each group independently. An important property of these data is that they may change over time due to the changes of attributes, and appearance/disappearance of users. Moreover, users may change their opinion about the targeted entity.

An ordinary clustering method is unlikely to adapt with such temporal dynamics of the data because it does not consider any relevant information, such as history and temporal effects. The notion of evolutionary clustering (Chakrabarti et al., 2006) appears in such situation, where the method should be specialized in clustering temporal data by taking care of the historic information and current data altogether. Numerous methods exist, which address these issues appropriately and cluster temporal data. These methods are based on different strategies, such as spectral clustering (Chi et al., 2009; Xu et al., 2014) and probabilistic generative model (Blei and Lafferty, 2006; Xu et al., 2012; Kim et al., 2015). However, it remains an important issue - how to interpret the evolution of the clusters. In this research, we are motivated by this issue and propose a novel method based on the Multinomial mixture model (Bishop et al., 2006) to cluster the temporal data as well as interpret the evolution of the clusters through some prior belief. Therefore, we propose a novel method which simultaneously performs evolutionary clustering and interpret the evolution.

\footnotetext{
*Corresponding author. Address: Laboratoire ERIC, 5 av. Mendès France 69676 BRON Cedex, FRANCE; Tel.: 0033478772609.

Email addresses: mhasnat@gmail.com (Md. Abul Hasnat), julien.velcin@univ-lyon2.fr (Julien Velcin), stephane.bonnevay@univ-lyon1.fr (Stephane Bonnevay), julien.jacques@univ-lyon2.fr (Julien Jacques)
} 
Multinomial Mixture (MM) model based clustering strategy is a popular method for clustering discrete data (Meilă and Heckerman, 2001; Agresti, 2002). Most recently, it has been exploited to perform evolutionary clustering (Kim et al., 2015). In this research, we consider MM as the core model for the data and propose an evolutionary clustering method by deriving appropriate link between the parameters of MM at different time.

Parametric link among probability distributions has been used in the context of transfer learning (Biernacki et al., 2002; Beninel et al., 2012), where the goal is to adapt a clustering model from a source population to a target one. In the context of continuous features, Biernacki et al. (2002) proposed a parametric link between the Normal distributions. Jacques and Biernacki (2010) extended it for the binary features using the Bernoulli distribution. However, no such formulation exists for the Multinomial distribution. Moreover, such parametric link-based methods are never considered in the context of evolutionary clustering. We are motivated from both of these issues and propose a clustering method that exploits the links among the parameters of the Multinomial distributions to analyze the temporal/evolutionary data.

Our overall contribution in this research is to propose a novel evolutionary clustering method based on the Multinomial mixture model. The highlights of our contributions include: (a) propose a formulation for parametric link among the Multinomial distributions; (b) develop a novel evolutionary clustering method by exploiting the link parameters and (c) provide interpretation of the link parameters to describe cluster evolution. First, we use synthetic data to evaluate and compare the proposed method w.r.t. the state-ofthe-art methods. Next, we apply it to analyze the temporal dynamics of social media data obtained from the ImagiWeb project (Velcin et al., 2014). Results in Sec. 4 show that the proposed method is better than the state-of-the-art methods.

In the rest of the paper, we provide related background in Sec. 2, describe our proposed method in Sec. 3, present the experimental results and observations in Sec. 4 and finally draw conclusions in Sec. 5 .

\section{Background and related work}

Evolutionary Clustering (ECL), also called clustering over time, aims to cluster the data that dynamically evolves over time (Chakrabarti et al., 2006). ECL methods cluster the data by considering the temporal smoothness to reflect the long-term trends of the data while being robust to the short-term variations. The demand and applications of these methods are increasing rapidly in various domains. They have been successfully applied to analyze news (Xu et al., 2012), social media (Kim et al., 2015), stock price (Xu et al., 2014), photo-tag pairs (Chakrabarti et al., 2006) and documents (Blei and Lafferty, 2006).

Temporal/evolutionary data clustering has been addressed from several viewpoints in the literature, which naturally raises several task-specific notions about ECL. A distinction among them can be as follows: (1) clustering; (2) monitoring and (3) interpreting. In the following paragraphs, we review relevant work based on this distinction.

Following the definition of Chakrabarti et al. (2006), the ECL method clusters data by considering the historic and current information. Based on this definition, we do not consider the methods which do not take into account the historic information. Besides, in order to limit our focus on the parametric methods, we do not consider the methods from non-parametric Bayesian based approaches (Xu et al., 2008; Dubey et al., 2013; Kharratzadeh et al., 2015).

Numerous ECL methods have been proposed in the literature. Chakrabarti et al. (2006) provided a generic framework and proposed different versions with the k-means and hierarchical clustering. It is based on optimizing a global cost function, which incorporates the snapshot (static clustering) quality and history cost (temporal smoothness). Chi et al. (2009) proposed two methods based on spectral clustering. They added different terms within the cost functions to regularize the temporal smoothness. Xu et al. (2014) recently proposed AFFECT, which performs adaptive evolutionary clustering by estimating an optimal smoothing parameter. It is extended with several static methods, such as k-means, hierarchical and spectral. A common property of these methods is that they are specialized for continuous data. Therefore, they may not be an appropriate choice for the categorical data, which is our concern in this research.

Dynamic Topic Model (DTM) is a well-known method for analyzing temporal categorical data (Blei and Lafferty, 2006). It extends the popular topic modeling method called Latent Dirichlet Allocation (LDA) 
(Blei et al., 2003). It uses Dirichlet prior based smoothing, which sometime over-smooth the data. As a consequence, it may cluster the data samples with non-co-occurring features in the same group (Kim et al., 2015). This causes DTM to underperform when clustering the classical non-textual temporal categorical data. Recently, Kim et al. (2015) address this issue and proposed Temporal Multinomial Mixture (TMM). TMM extends the classical Multinomial mixture (MM) model by incorporating temporal dependency into the relation between the data of current time epoch and the clusters of the previous time epoch. Indeed, TMM is more related to our proposed approach as we aim to establish parametric link among MMs at different time epochs. Unfortunately, both DTM and TMM are unable to detect and interpret cluster evolution, which is one of the main foci of this research.

Evolution monitoring (Spiliopoulou et al., 2006) tracks the clusters evolution by identifying the birth, death, split, merge and survival of clusters at different time. An external clustering method is first used at each time, e.g., Spiliopoulou et al. (2006) and Oliveira and Gama (2010) used the k-means method, whereas Lamirel (2012) used the neural clustering method. Afterward, the mapping among the clusters at different time is examined based on several heuristics. A different method, called label-based diachronic approach (Lamirel, 2012), exploits the MultiView Data Analysis technique among the cluster labels at different time. This approach constructs heuristics from features for monitoring cluster evolution. Our approach is different than the above methods, because: (a) we do not aim to propose a cluster monitoring method explicitly and (b) we do not use a static clustering method. Ferlez et al. (2008) proposed a joint clustering-monitoring method which uses the cross association algorithm to cluster data and a bipartite graph to monitor evolution. They group the distinct features (word) in each cluster and hence features do not coexist in different clusters. This is different than us as we exploit all the features in order to provide a feature level interpretation for the evolution.

Evolution interpretation aims to explain the reason for the evolution of clusters at different time. It can be accomplished by explicitly analyzing the features. Lamirel (2012) used the F-measures from individual features and constructs a similarity report. We obtain the interpretation directly from the link parameters, which are estimated as a part of clustering. Therefore, unlike Lamirel (2012), we do not need any external analysis of the features.

Based on the above distinctions we find that our method is more similar to evolutionary clustering than evolution monitoring. Therefore, we compare it only with the relevant methods, such as Xu et al. (2014), Blei and Lafferty (2006) and Kim et al. (2015).

Now we focus on the literature related to our proposal. The idea of parametric link in a transfer learning context (Beninel et al., 2012) is inherited from the concept for Generalized Discriminant Analysis (GDA) (Biernacki et al., 2002). GDA adapts the classification rule from a source population to a target population through a linear link map of their parameters. Biernacki et al. (2002) proposed several models for GDA within the context of multivariate Gaussian distribution. Later, Jacques and Biernacki (2010) extend it for binary data using the Bernoulli distribution (Bishop et al., 2006). We observe that, these approaches can be exploited to develop an evolutionary clustering method by replacing the notion of source/target with different time epochs $t-1 / t$. Besides, such development requires the derivation of the linear link for the Multinomial distribution.

The Multinomial distribution is a standard probability distribution for analyzing the discrete categorical data (Agresti, 2002). The Multinomial Mixture (MM) is a statistical model based on the Multinomial distribution. It has been used for cluster analysis with discrete data (Meilă and Heckerman, 2001; Zhong and Ghosh, 2005; Hasnat et al., 2015). Meilă and Heckerman (2001) studied several Model-Based Clustering (MBC) methods with the MM and experimentally compared them using different criteria such as clustering accuracy, computation time and number of selected clusters. Silvestre et al. (2014) proposed a MBC method, which integrates both model estimation and selection within a single EM algorithm. Recently, Hasnat et al. (2015) proposed a MBC method which performs simultaneous clustering and model selection using the MM. Their strategy performs similar task as Silvestre et al. (2014) in a computationally efficient manner, which has been previously proposed for the Gaussian distribution (Garcia and Nielsen, 2010) and the Fisher distribution (Hasnat et al., 2016). Following the above methods, we exploit the MBC framework.

MBC (Fraley and Raftery, 2002; Melnykov and Maitra, 2010) is a well-established method for cluster analysis and unsupervised learning. It assumes a probabilistic model (e.g., mixture model) for the data, 
estimates the model parameters by optimizing an objective function (e.g., model likelihood) and produces probabilistic clustering. The Expectation Maximization (EM) (McLachlan and Krishnan, 2008) is mostly used in MBC to estimate the model parameters. EM consists of an Expectation step (E-step) and a Maximization step (M-step), which are iteratively employed to maximize the log-likelihood of the data. Initialization of the EM algorithm has significant impact on clustering results (McLachlan and Krishnan, 2008; Baudry and Celeux, 2015). With different initializations the EM algorithm may converge to different values of the likelihood function, some of which can be local maxima (i.e., sub-optimal results). In order to overcome this, numerous different initialization strategies are proposed and experimented in the relevant literature (Biernacki et al., 2003; Meilă and Heckerman, 2001; Baudry and Celeux, 2015; Hasnat et al., 2015). Following recommendations, we use the small-EM (Biernacki et al., 2003, 2006; Baudry and Celeux, 2015; Hasnat et al., 2015) method to initialize the MM parameters.

MBC strategies have been commonly exploited to identify the best model for the data by fitting a set of models with different parameterizations and/or number of components and then applying a statistical model selection criterion (Fraley and Raftery, 2002; Biernacki et al., 2000; Figueiredo and Jain, 2002; Melnykov and Maitra, 2010; Hasnat et al., 2016). In this paper, we apply this model fitting and selection strategy for two purposes: (a) to identify the parametric sub-models (Section 3.3) and (b) to automatically select the number of components (Section 3.6).

\section{Parametric Link Based Evolutionary Clustering}

We adopt the parametric link approach (Biernacki et al., 2002; Jacques and Biernacki, 2010) for evolutionary clustering by assuming that the source samples are equivalent to the samples at time epoch $t$ and the target samples represent the samples of time $t+1$. With this assumption, we incorporate a linear link between the Multinomials at different time epochs. The algorithm for the proposed clustering method is presented in Algorithm 1.

\subsection{Statistical model for evolutionary data samples}

Let $S^{t}$ be a set of samples corresponding to time $t$ and $S^{t+1}$ be a set from the next time $t+1$. We assume that while the cluster labels for $S^{t}$ are known to us (estimated from $t-1$ ), labels of $S^{t+1}$ are unknown.

Let $S^{t}$ be composed of $N^{t}$ pairs $\left(\mathbf{x}_{1}^{t}, \mathbf{z}_{1}^{t}\right), \ldots,\left(\mathbf{x}_{N^{t}}^{t}, \mathbf{z}_{N^{t}}^{t}\right)$, where $\mathbf{x}_{i}^{t}=\left\{x_{i, 1}^{t}, \ldots, x_{i, D}^{t}\right\}$ is the $D$ dimensional count vector of order $V$, i.e., $\sum_{d=1}^{D} x_{i, d}^{t}=V . \mathbf{z}_{i}^{t}$ is the associated class label such that $\mathbf{z}_{i, k}^{t}=1$ if the data belongs to cluster $k$ with $k=1, \ldots, K$ and $\mathbf{z}_{i, k}^{t}=0$ otherwise. We assume that any sample $\mathbf{x}_{i}^{t}$ of $S^{t}$ is an independent realization of the random variable $\mathbf{X}^{t}$ of distribution:

$$
\mathbf{X}^{t} \sim \mathcal{M}\left(V, \boldsymbol{\mu}_{k}^{t}\right), \quad k=1, \ldots, K
$$

with $\mathcal{M}\left(V, \boldsymbol{\mu}_{k}^{t}\right)$ is the $V$-order Multinomial distribution with parameter $\boldsymbol{\mu}_{k}^{t}=\left(\mu_{k, 1}^{t}, \ldots, \mu_{k, D}^{t}\right)$, which is formally defined as (Bishop et al., 2006) (in order to avoid redundancy, we do not use the superscript $t$ for the notations in the generalized equations, such as (3.1, 3.2, 3.6, 3.7 and 3.8); Because, the definitions and derivations in these equations are time independent, i.e. remains same for any time instance $t$ ):

$$
\mathcal{M}\left(\mathbf{x}_{i} \mid V, \boldsymbol{\mu}_{k}\right)=\left(\begin{array}{c}
V \\
x_{i, 1}, x_{i, 2}, \ldots, x_{i, D}
\end{array}\right) \prod_{d=1}^{D} \mu_{k, d}^{x_{i, d}}
$$

here, $\boldsymbol{\mu}_{k}$ is the parameter of the Multinomial distribution of $k^{t h}$ class with $0 \leq \mu_{k, d} \leq 1$ and $\sum_{d=1}^{D} \mu_{k, d}=1$. Therefore, samples of the entire set $S^{t}$ can be modeled with a mixture of $K$ Multinomials, also called Multinomial Mixture (MM) model, which has the following form:

$$
f\left(\mathbf{x}_{i} \mid \Theta_{K}\right)=\sum_{k=1}^{K} \pi_{k} \mathcal{M}\left(\mathbf{x}_{i} \mid V, \boldsymbol{\mu}_{k}\right)
$$


In Eq. (3.2), $\Theta_{K}=\left\{\left(\pi_{1}, \boldsymbol{\mu}_{1}\right), \ldots,\left(\pi_{K}, \boldsymbol{\mu}_{K}\right)\right\}$ is the set of model parameters, $\pi_{k}$ is the mixing proportion with $\sum_{k=1}^{K} \pi_{k}=1$ and $\mathcal{M}\left(\mathbf{x}_{i} \mid V, \boldsymbol{\mu}_{k}\right)$ is the density function (Eq. (3.1)). Besides, we assume that the class label $\mathbf{z}_{i}^{t}$ is an independent realization of a random vector $\mathbf{Z}^{t}$, distributed according to 1-order Multinomial:

$$
\mathbf{Z}^{t} \sim \mathcal{M}\left(1, \boldsymbol{\pi}^{t}\right)
$$

where $\boldsymbol{\pi}^{t}=\pi_{1}^{t}, \ldots, \pi_{K}^{t}$ is the mixing proportion of the model in Eq. (3.2).

The assumption of MM is similar for the samples of $S^{t+1}$ with random variable $\mathbf{X}^{t+1}$ and parameter $\boldsymbol{\mu}_{k}^{t+1}$. However, for $S^{t+1}$ the labels $\mathbf{z}_{i}^{t+1}$ of $N^{t+1}$ pairs $\left(\mathbf{x}_{1}^{t+1}, \mathbf{z}_{1}^{t+1}\right), \ldots,\left(\mathbf{x}_{N^{t+1}}^{t+1}, \mathbf{z}_{N^{t+1}}^{t+1}\right)$ are unknown. In the context of evolutionary clustering, our goal is to estimate the unknown labels $\mathbf{z}_{i}^{t+1}$ for $i=1, \ldots, N^{t+1}$ using the information from $S^{t}$ and $S^{t+1}$ by establishing a link between $\boldsymbol{\mu}_{k}^{t}$ and $\boldsymbol{\mu}_{k}^{t+1}$.

Identifiability of mixture of Multinomials. It is known that mixture of Multinomials are strictly not identifiable and Allman et al. (2009) give the generic conditions for model identifiability. This condition needs that the number of variables $(D)$ is significantly bigger than the number of clusters $(K)$, which is the case in many data sets and especially in the Twitter data studied in this paper.

\subsection{Parametric link/relationship among temporal data}

For random variables $Y^{t}$ and $Y^{t+1}$ distributed according to the Gaussian distribution, a linear distributional link exists (under weak assumptions) (Biernacki et al., 2002). It has the form: $Y^{t+1} \sim D Y^{t}+b$, where $D$ and $b$ are the link parameters among the samples of different time epochs. For binary data, the following distributional linear link among the Bernoulli parameters $\left(\alpha^{t+1}\right.$ and $\alpha^{t}$ with $\left.0 \leq \alpha \leq 1\right)$ is derived by Jacques and Biernacki (2010):

$$
\alpha^{t+1}=\Phi\left(\delta \Phi^{-1}\left(\alpha^{t}\right)+\lambda \gamma\right)
$$

where $\delta \in \mathbb{R}^{+} \backslash\{0\}, \lambda \in\{-1,1\}$ and $\gamma \in \mathbb{R}$ are the link parameters. $\Phi$ is the cumulative Gaussian function of mean 0 and variance 1 . Note that this link is not identifiable. The sub-models as well as constraints on the model parameters are given in Jacques and Biernacki (2010) in order to define identifiable model for the link between two time epochs. Unfortunately, we cannot directly apply Eq. (3.3) for the Multinomial distribution, because it violates the constraint on the parameter $\mu$. However, we can modify the above formulation by considering two issues: (1) Multinomial parameter $\boldsymbol{\mu}_{k}$ has similar property as $\boldsymbol{\alpha}_{k}$ except $\sum_{d=1}^{D} \mu_{k, d}=1$ and (2) samples from $X$ are not necessary to be binary, which makes $\lambda$ as an unnecessary variable (it was introduced in Jacques and Biernacki (2010) to handle binary observations). Considering these issues, we can derive the parametric link between $\boldsymbol{\mu}^{t}$ and $\boldsymbol{\mu}^{t+1}$ as:

$$
\mu_{k, d}^{t+1}=\frac{\Phi\left(\delta_{k, d} \Phi^{-1}\left(\mu_{k, d}^{t}\right)+\gamma_{k, d}\right)}{\sum_{r=1}^{D} \Phi\left(\delta_{k, r} \Phi^{-1}\left(\mu_{k, r}^{t}\right)+\gamma_{k, r}\right)}
$$

where $\delta_{k, d} \in \mathbb{R}^{+} \backslash\{0\}$ and $\gamma_{k, d} \in \mathbb{R}$ are the link parameters. In Eq. (3.4), the combination of parameters $\delta_{k, d}$ and $\gamma_{k, d}$ for $\forall k, d$ is called a full model which is over-parameterized. Instead, we consider several sub-models with certain constraints on the parameters, prohibiting for instance $\delta_{k, d}$ and $\gamma_{k, d}$ to be free together. The main idea is to define a family of sub-models from very parsimonious sub-models to more complex ones.

Notation. At each time epoch, the parameters $\delta_{k, d}$ and $\gamma_{k, d}$ express the link between the past $(t-1)$ and present $(t)$ time changes, and they should be noted $\delta_{k, d}^{t}$ and $\gamma_{k, d}^{t}$. Nevertheless, for the sake of notation simplicity, the superscript is omitted.

\subsection{Parametric sub-models}

The idea of defining sub-models is frequent in Model-Based Clustering (MBC) (Fraley and Raftery, 2002). We fit the evolutionary clustering model (Eq. (3.4)) with different sub-models and then select the best model using the Bayesian Information Criteria (BIC) (Schwarz et al., 1978):

$$
B I C=-2 L(\Theta)+\nu \log \left(N^{t+1}\right)
$$


where $L(\Theta)$ is the log-likelihood (Eq. (3.6)) value associated to the MM parameters of time $t+1, \nu$ is the number of free parameters of the sub-model. These sub-models provide sufficient interpretation about the change in parameters from time $t$ to $t+1$. Definition and interpretation of several basic sub-models, defined as pair $\left(\delta_{k, d} / \gamma_{k, d}\right)$ are given below:

(M1) $1 / 0$ : This model is constrained with $\delta_{k, d}=1$ and $\gamma_{k, d}=0$ for $\forall k, d$, i.e., $\nu=0$. It indicates that the observations $X^{t+1}$ can be modeled with $\mu_{k, d}^{t}$ and hence no evolution occurred.

(M2) $0 / \gamma_{k, d}$ : This model is constrained with $\delta_{k, d}=0$ for $\forall k, d$, i.e., $\nu=K * D$. It indicates that the observations $X^{t+1}$ should be modeled without considering $\mu_{k, d}^{t}$. This model should be selected when a new cluster evolved independently and does not consider any historical information. This is the most general model that can efficiently fit the observations $X^{t+1}$ to a MM model, subject to a good initialization of the alternative iterative method. Several possible variations of this model are: $0 / \gamma, 0 / \gamma_{k}$ and $0 / \gamma_{d}$. In these notations, subscript $k$ means cluster dependent and $d$ means feature dependent, and no subscription means a constant value for all clusters and features).

(M3) $\delta_{k, d} / 0$ : This model is constrained with $\gamma_{k, d}=0$ for $\forall k, d$, i.e., $\nu=K * D$. It indicates that $\mu_{k, d}^{t+1}$ are evolved through $\mu_{k, d}^{t}$ in a specific transformation space (inversed cumulative Gaussian). This model should be selected when true evolution occurred, which can be explained in detail through a certain belief on the observed features and obtained clusters. Moreover, such a model can be plugged-in independently with any external clustering method in order to describe the evolution. Several possible variations of this model are: $\delta / 0, \boldsymbol{\delta}_{k} / 0$ and $\boldsymbol{\delta}_{d} / 0$. This model is equivalent to the fundamental unconstrained model assumed by Biernacki et al. (2002).

(M4) $1 / \gamma_{k, d}$ : In this model, $\delta_{k, d}=1$ for $\forall k, d$, i.e., $\nu=K * D$. This model does nearly similar task as model M3. It is relatively easier to fit through the additive term in the inverse cumulative Gaussian space. On the other hand, it is less expressive in terms of interpretation. Several possible variations of this model are: $1 / \gamma, 1 / \gamma_{k}$ and $1 / \gamma_{d}$.

Sub-models identifiability. In the case of binary data, Jacques and Biernacki (2010) consider similar submodels for the link parameters and prove that these sub-models are identifiable (at least in practice). In the Multinomial case, the parameters obtained by transformation (3.3) should be normalized in order to ensure that the Multinomial parameters $\mu_{k, d}$ sum (over $d$ ) to 1 . Thus, all of the models with feature-dependent link parameters (i.e. depending on $d$ ) are non identifiable. For the other sub-models, the identifiability is not proven in this paper and could be the subject of a future work. Nevertheless, we can mention that no identifiability problem has been observed in practice.

On the one hand, for some sub-models, several sets of link parameters can explain the same evolution, and thus the interpretation of the link parameters should be done carefully. On the other hand, the Multinomial parameters (which are identifiable), the sub-model choice and the clustering results can be interpreted without restriction, see Section 4.2.2 for an example of such interpretation for twitter data analysis.

\subsection{Parameter estimation}

In our proposed formulation of evolutionary clustering, we estimate two different types of parameters (see Eq. (3.4)): (1) MM model parameters: $\mu$ and $\pi$ and (2) temporal link parameters: $\delta$ and $\gamma$. We estimate them in two steps. The first step consists of estimating $\mu$ and $\pi$ (only for $t=1$ ) for the observed samples of time $t$. In the second step, we estimate $\delta$ and $\gamma$. At any time epoch, we estimate the class labels $\mathbf{z}_{i}$ by maximum a posteriori.

As discussed in Jacques and Biernacki (2010), it is possible to consider a full maximum-likelihood estimation of the model parameters for all time epochs, taking into account the whole sample of data. Such strategy is more efficient than our strategy in the case of small sample. However, it is equivalent if the sample size is sufficiently large, which is the case for the dataset studied in this paper. Moreover, the two steps strategy we propose can be easily used in an online system. Actually, the data for a new time epoch can be taken into account without estimating all the past model parameters. 


\subsubsection{Multinomial mixture parameters}

We estimate the MM parameters using an Expectation Maximization (EM) algorithm that maximizes the log-likelihood value which has the following form:

$$
L(\Theta)=\sum_{i=1}^{N} \log \sum_{j=1}^{K} \pi_{j} \mathcal{M}\left(\mathbf{x}_{i} \mid \boldsymbol{\mu}_{j}\right)
$$

where $N$ is the number of samples. In the Expectation step (E-step), we compute the posterior probability as:

$$
\rho_{i, k}=p\left(z_{i, k}=1 \mid \mathbf{x}_{i}\right)=\frac{\pi_{k} \prod_{d=1}^{D} \mu_{k, d}^{x_{i, d}}}{\sum_{l=1}^{K} \pi_{l} \prod_{d=1}^{D} \mu_{l, d}^{x_{i, d}}}
$$

In the Maximization step (M-step), we update $\pi_{k}$ and $\mu_{k, d}$ as:

$$
\pi_{k}=\frac{1}{N} \sum_{i=1}^{N} \rho_{i, k} \quad \text { and } \quad \mu_{k, d}=\frac{\sum_{i=1}^{N} \rho_{i, k} \mathbf{x}_{i, d}}{\sum_{i=1}^{N} \sum_{r=1}^{D} \rho_{i, k} \mathbf{x}_{i, r}}
$$

The E and M steps are iteratively employed until certain convergence criterion (difference of the log-likelihood values of successive iterations) is satisfied. The estimation of $\mu_{k, d}$ using Eq. (3.8) is only applicable for $t=1$ due to the unavailability of any temporal information. For any time $t+1$, when the link parameters are available, $\mu_{k, d}$ is estimated with Eq. (3.4).

\subsubsection{Link parameters}

Estimation of the link parameters $\delta_{k, d}$ and $\gamma_{k, d}$ uses $\mu_{k, d}^{t}$ and the observed samples at time $t+1$. Similar to Jacques and Biernacki (2010), we use again an EM algorithm, but in which the M step is not explicit. Consequently, we employ an external optimization method, such as an alternative iterative algorithm which consists of a succession, componentwise of the simplex method (Nelder and Mead, 1965). For the implementation, we used neldermead function of the nloptr R package (Ypma, 2014). The lower and upper bounds were set to -2.5 and +2.5 respectively only for the $\gamma_{k, d}$ parameters. In general, the starting point of the alternative algorithm corresponds to the case when $\mu_{k, d}^{t+1}=\mu_{k, d}^{t}$, i.e., $\delta_{k, d}=1$ and $\gamma_{k, d}=0$. However, in order to obtain a better estimate and save computation time (the simplex method requires a large number of iterations to converge), we apply an efficient approach, see Section 3.5.2.

\subsection{Parameters initialization}

In the proposed clustering method (Algorithm 1), we need to initialize both the MM parameters $\Theta_{K}^{\text {init }}=$ $\left\{\left(\pi_{1}^{i n i t}, \boldsymbol{\mu}_{1}^{i n i t}\right), \ldots,\left(\pi_{K}^{i n i t}, \boldsymbol{\mu}_{K}^{i n i t}\right)\right\}$ for time $t 1$ and the link parameters $(\delta$ and $\gamma)$.

\subsubsection{Multinomial mixture parameters}

Generally, the MM parameters are initialized randomly (Meilă and Heckerman, 2001; Hasnat et al., 2015). However, with both synthetic and real data it has been demonstrated by Hasnat et al. (2015) that, random initialization has its limitation w.r.t. the clustering performance and stability. Therefore, following Hasnat et al. (2015), we initialize the model parameters using the small-EM procedure. It consists of running multiple short runs of randomly initialized EM and then selecting the one with the maximum-likelihood value. Here, short run means the EM procedure does not need to wait until convergence and it can be stopped when a certain number of iterations is completed.

\subsubsection{Link parameters}

We propose an initialization procedure based on the predictive parameters set for next time epoch $\Theta_{K}^{\text {pred }}=\left\{\left(\pi_{1}^{\text {pred }}, \boldsymbol{\mu}_{1}^{\text {pred }}\right), \ldots,\left(\pi_{K}^{\text {pred }}, \boldsymbol{\mu}_{K}^{\text {pred }}\right)\right\}$. Let $\Theta_{K}^{t}=\left\{\left(\pi_{1}^{t}, \boldsymbol{\mu}_{1}^{t}\right), \ldots,\left(\pi_{K}^{t}, \boldsymbol{\mu}_{K}^{t}\right)\right\}$ is the set of parameters for the current time $(t)$ epoch. Our initialization procedure consists of the following steps: 


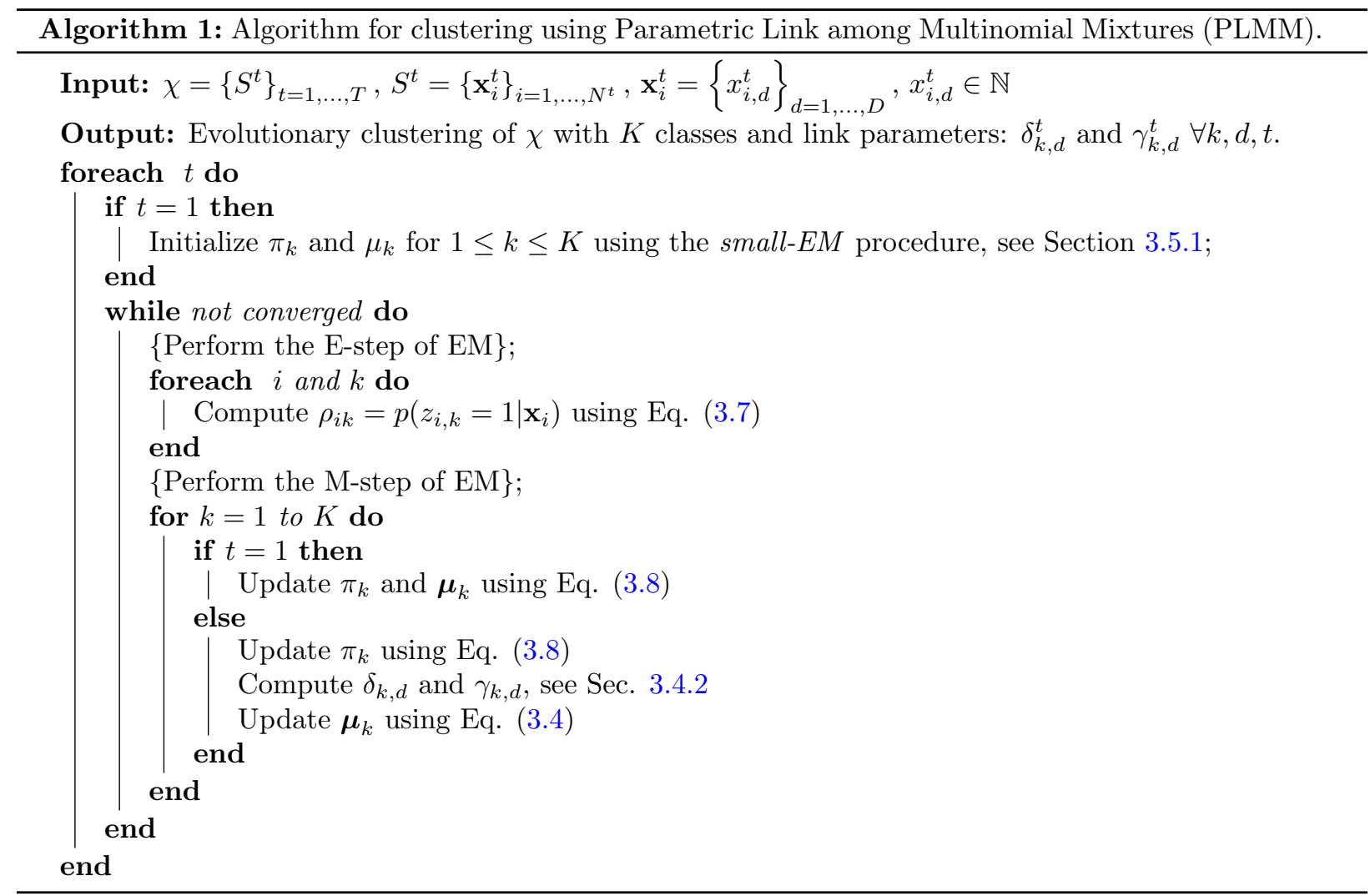

- Step 1: estimate $\Theta_{K}^{\text {pred }}$ using data samples of next time $X^{t+1}$ and an EM algorithm which is initialized with $\Theta_{K}^{t}$.

- Step 2: compute $\delta_{k, d}^{\text {init }}$ and $\gamma_{k, d}^{\text {init }}$ for each $k$ and $d$ as:

$$
\begin{gathered}
\gamma_{k, d}^{\text {init }}=\Phi^{-1}\left(\mu_{k, d}^{\text {pred }}\right) \quad \text { for model M2 } \\
\delta_{k, d}^{\text {init }}=\frac{\Phi^{-1}\left(\mu_{k, d}^{\text {pred }}\right)}{\Phi^{-1}\left(\mu_{k, d}^{t}\right)} \quad \text { for model M3 } \\
\gamma_{k, d}^{\text {init }}=\Phi^{-1}\left(\mu_{k, d}^{\text {pred }}\right)-\Phi^{-1}\left(\mu_{k, d}^{t}\right) \quad \text { for model M4 }
\end{gathered}
$$

The Eq. (3.9), (3.10) and (3.11) are simply derived from Eq. (3.4) with the consideration that denominator is equal to 1 , i.e., $\sum_{d=1}^{D} \mu_{k, d}=1$ for $k=1, \ldots, K$.

\subsection{Varying number of clusters}

The methodology presented in the previous sub-sections assumes the same number of clusters $K$ for each time epoch. In this sub section, we propose an extension of it such that the method can handle varying $K$ at different time, i.e., $K^{t}$ and $K^{t+1}$ may be different. We modify the link parameters initialization strategy (Section 3.5.2) to adapt the variability among $\Theta_{K^{t}}^{t}$ and $\Theta_{K^{t+1}}^{t+1}$. At time epoch $t$, this extended method requires additional information, such as: (a) number of clusters $K^{t+1}$ and (b) cluster mapping between $\Theta_{K^{t}}^{t}$ and $\Theta_{K^{t+1}}^{t+1}$. 
We adopted the EM-HAC method proposed by Hasnat et al. (2015) with the L-method Salvador and Chan (2004) to select the number of cluster automatically at each time epoch. This approach consists of first generating a set of MM models with different values of $K\left(2 \ldots K_{\max -1}\right)$. Then it constructs a plot based on the BIC (Eq. 3.5) values computed from the MM models of the set. This plot consists of the number of clusters in its $x$-axis and associated BIC values in its $y$-axis. Finally, the optimal number of clusters is selected by detecting the knee point in the plot as follows: (a) fit two lines at the left and right side of each point in the $x$-axis within the range $2, \ldots, K_{\max }-1$; (b) compute the total weighted root mean squared error (RMSE) for fitting lines at each point and (c) select the point with lowest RMSE value.

In order to initialize the link parameters, first we select the number of clusters $K^{t+1}$ and obtain the set of predictive parameters $\Theta_{K^{t+1}}^{\text {pred }}$. Next, for each cluster $k$ in $\Theta_{K^{t+1}}^{\text {pred }}$, we find the corresponding cluster in $\Theta_{K^{t}}^{t}$ based on the minimum symmetric Kullback Leibler divergence (sKLD). sKLD among two clusters $a$ and $b$ is defined as (Hasnat et al., 2015):

$$
\begin{array}{r}
s K L D=\frac{D_{K L}\left(\boldsymbol{\mu}_{a}, \boldsymbol{\mu}_{b}\right)+D_{K L}\left(\boldsymbol{\mu}_{b}, \boldsymbol{\mu}_{a}\right)}{2}, \text { where } \\
D_{K L}\left(\boldsymbol{\mu}_{a}, \boldsymbol{\mu}_{b}\right)=\sum_{d=1}^{D} \mu_{a, d} \ln \left(\frac{\mu_{a, d}}{\mu_{b, d}}\right)
\end{array}
$$

After establishing the correspondences, we use Eq. (3.9), (3.10) and (3.11) to set the initial values of the link parameters. Finally, we estimate the link parameters following Section 3.4.2.

\subsection{Interpretation of cluster evolution}

The link parameters $\left(\delta_{k, d}\right.$ and $\left.\gamma_{k, d}\right)$ along with the function $\Phi$ are the key to interpret the cluster evolution. First of all, let us recall that some of the sub-models are not identifiable (see end of Section 3.3), what means that several sets of link parameters can explain the same evolution. In particular, this is the case of all models having link feature-dependent parameters (i.e. depending on $d$ ). Consequently, several interpretations of the clustering evolution can co-exist. That being said, let us notice some basic interpretation of the values of these parameters for all feature $d$ and cluster $k$ :

- $\delta_{k, d}=0$ means that $\mu_{k, d}$ (probability) at $t+1$ does not depend on $t$, whereas $\delta_{k, d}=1$ (with $\left.\gamma_{k, d}=0\right)$ means identical probability at two different times.

- $\delta_{k, d} \rightarrow 0$ and/or $\gamma_{k, d} \rightarrow \infty$ means that the distribution tends to uniform distribution.

- $\delta_{k, d} \rightarrow \infty$ and/or $\gamma_{k, d} \rightarrow-\infty$ means that the distribution tends to be more concentrated (Dirac distribution) at time $t+1$ in the feature which has the highest probability at time $t$.

In order to get further interpretation, we need to understand the Multinomial parameters $\mu_{k, d}$ and the space spanned by the cumulative Gaussian $\Phi$ and its inverse $\Phi^{-1}$. Let us consider an experiment of drawing $V$ balls of $d=1, \ldots, D$ different colors (represent features). After each draw, the color of the ball is recorded in a $D$ dimensional count vector $\mathbf{x}_{i}$ and the ball is replaced. Therefore, at the end of $i^{\text {th }}$ experiment $\mathbf{x}_{i, d}$ reveals the count of drawing the $d^{t h}$ colored ball. When a Multinomial distribution is used to fit such experimental data, its parameter $\mu_{k, d}$ reveals the probability of drawing the $d^{\text {th }}$ colored ball.

Now let us consider $\Phi$ in Fig. 3.1, where the values along the Y-axis represent the possible values of $\mu_{k, d}^{t+1}$ (with $0 \leq \mu_{k, d}^{t+1} \leq 1$ ) and the X-axis represents the values of $\mu_{k, d}^{t}$ after transforming through $\Phi^{-1}$ function. Now, according to Eq. (3.4), cluster evolutions $\left(\mu_{k, d}^{t} \rightarrow \mu_{k, d}^{t+1}\right)$ can be explained through multiplication (using $\delta_{k, d}$ ) and addition/subtraction (using $\gamma_{k, d}$ ) operations.

The values of $\gamma_{k, d}$ can certainly indicates the increase/decrease of the probability of certain feature (color) subject to the selection of sub-model M4. On the other hand if sub-model M3 is selected, values of $\delta_{k, d}$ can explain the belief that $\mu_{k, d}^{t+1}$ should decrease if $\mu_{k, d}^{t}<0.5$ and increase if $\mu_{k, d}^{t}>0.5$. For example, let us consider that in a 2 colors (red and green) ball experiment the probability of the red color ball is changed from 0.8 (at time t1) to 0.7 (at time t2). Such a change can be explained with model M3 with $\delta_{k, \text { red }}=0.6$, which indicates that the belief is decreased at the next time. From the above discussions it is evident that the proposed method is capable to interpret the cluster evolutions up to the feature level. 


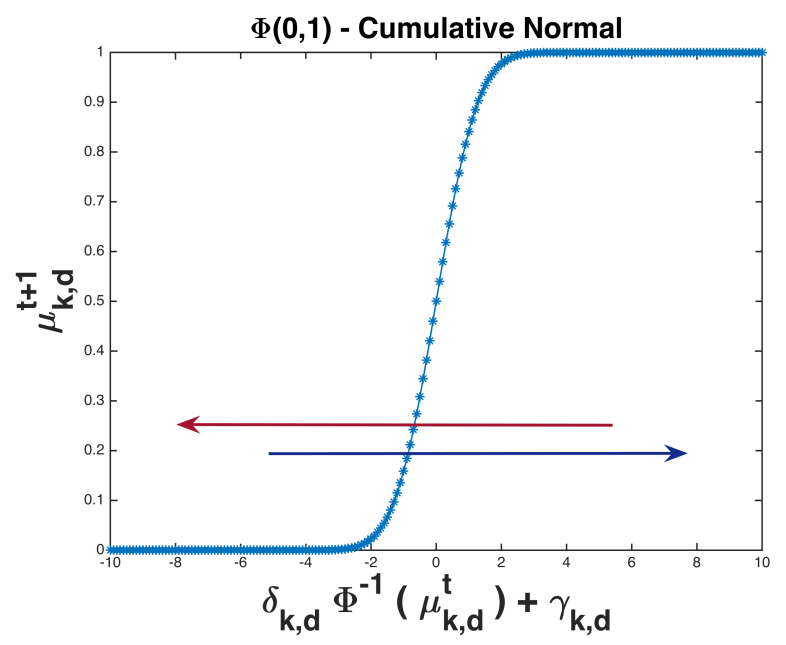

Figure 3.1: Illustrations of Cumulative Gaussian function and its relationship with the parameter change of Multinomial distribution using Eq. (3.4). The arrows indicates the direction of changes in the inverse function space which eventually increase/decrease the probability.

\section{Numerical experiments}

We begin the experiments using the simulated evolutionary data samples and evaluate w.r.t. the state-ofthe-art methods. A characteristic comparison of different methods is presented in Table 1. For the simulated samples, we use the Accuracy and Adjusted Rand Index (ARI) (Hubert and Arabie, 1985) as the measures for evaluation. Next, we experiment and compare methods using the real data. We use one of the real datasets experimented by Kim et al. (2015). We choose the political opinion dataset from the ImagiWeb project (Velcin et al., 2014) as it consists of data from an interesting time period - during and after the election.

Table 1: Characteristic comparison of different state-of-the-art evolutionary clustering methods: Parametric Link among Multinomial Mixtures (PLMM, our proposed method), Temporal Multinomial Mixture (TMM) (Kim et al., 2015), Dynamic Topic Model (DTM) (Blei and Lafferty, 2006) and adaptive evolutionary clustering method (AFFECT) (Xu et al., 2014).

\begin{tabular}{ccccc}
\hline & PLMM & DTM & TMM & AFFECT \\
\hline Data Type & Discrete & Discrete & Discrete & Continuous \\
\hline Interpret Evolution & Yes & No & No & No \\
\hline
\end{tabular}

\subsection{Simulated Data Samples}

Following the standard sampling methods, we generate different sets $\left\{S^{t}\right\}_{t=1, \ldots, T}$ of simulated data for different time epochs. We draw a finite set of categorical samples (discrete count vectors) $S^{t}=\left\{\mathbf{x}_{i}\right\}_{i, \ldots, N^{t}}$ with different dimensions of features $D$, such as 10, 20 and 40. These samples are issued from the Multinomial mixture (MM) models of $K=3$ classes. We consider two different sets of samples:

- Samples with higher order of categorical count (hos) with $V \sim 1.5 * D$ for 3 time epochs. Each epoch draws different number of i.i.d. samples: $N^{1}=500, N^{2}=100$ and $N^{3}=200$. We also add noisy counts with them. This type of samples provides better resemblance with the MM parameters due to sufficient number of count in the observations. Practically, this is similar to the fact when the observations consist of data over a longer period of time.

- Samples with lower order of categorical count (los) with $V \sim 0.7 * D$ for 5 time epochs. Each epoch draws different number of i.i.d. samples: $N^{1}=50, N^{2}=40, N^{3}=40, N^{4}=30$ and $N^{5}=20$. This 
type of samples are sparse and often difficult to distinguish among clusters. Practically, this is similar to the fact when the observations consist of data over a shorter period of time.

The evolutionary data generation process consists of two steps: (1) determine MM parameters $\mu_{k, d}$ at each time epoch $t=1, \ldots, T$ and (2) sample observations from the MM following the assumption specified by Blei et al. (2003). For $t=1$, we sample $\mu_{k, d}$ from a Dirichlet distribution and verify (separation w.r.t. the other clusters parameters (Silvestre et al., 2014)) it using the symmetric Kullback-Leibler Divergence value. For $t>1$, we sample $\mu_{k, d}$ from $\mu_{k, d}^{t-1}$ using the MM link relationship defined in Eq. (3.4). This ensures that, we maintain the temporal smoothness property (Chakrabarti et al., 2006) of the evolutionary data samples. In order to use the link relationship, first we randomly select a model and then set the associated link parameters $\left(\delta_{k, d}\right.$ and $\left.\gamma_{k, d}\right)$ within a pre-specified range of values.

To sample observations, first we choose the order $V_{k}$ of each cluster. Our sampling procedure for each observation $i$ at each time $t$ follows the steps below:

- Choose a cluster $z_{i, k}=1$ as: $\mathbf{z}_{i} \sim \mathcal{M}\left(1, \pi_{1}, \ldots, \pi_{D}\right)$, with, $\pi_{d}=\frac{1}{k}$.

- Choose the order $\tau_{i}$ of the Multinomial for $\mathbf{x}_{i}$ using the Poisson distribution as: $\tau_{i} \mid z_{i, k}=1 \sim$ Poisson $\left(V_{k}\right)$.

- Draw sample $\mathbf{x}_{i}$ using the Multinomial distribution as: $\mathbf{x}_{i} \mid \tau_{i}, z_{i, k}=1 \sim \mathcal{M}\left(\tau_{i}, \mu_{k, 1}, \ldots, \mu_{k, D}\right)$.

Table 2: Simulated data evaluation and comparison using the classification accuracy (in \%) and Adjusted Rand Index (ARI) (Hubert and Arabie, 1985). Methods: PLMM (proposed), Dynamic Topic Model (DTM), Temporal Multinomial Mixture (TMM) and AFFECT with k-means. Datasets consist of different types (hos and los) of samples with different numbers (10, 20 and 40) of features. hos: higher order samples and los: lower order samples. Boldfaced indicate the best result. Values for accuracy and ARI are separated by comma.

\begin{tabular}{ccccc}
\hline & PLMM & TMM & DTM & AFFECT \\
\hline 10, hos & $\mathbf{9 6 . 8 , 0 . 9 2}$ & $91.2,0.84$ & $90.7,0.79$ & $42.9,0.31$ \\
\hline 10, los & $\mathbf{9 7 . 1 , 0 . 9 3}$ & $93.7,0.88$ & $92.2,0.83$ & $46.3,0.34$ \\
\hline 20, hos & $\mathbf{9 8 . 8 , 0 . 9 6}$ & $93.6,0.88$ & $90,0.8$ & $44.2,0.23$ \\
\hline $\mathbf{2 0}$, los & $\mathbf{9 9 . 9 , 0 . 9 9}$ & $98.1,0.97$ & $94.4,0.92$ & $45.9,0.33$ \\
\hline 40, hos & $\mathbf{9 8 . 9 , 0 . 9 7}$ & $91.1,0.85$ & $69.4,0.46$ & $42.3,0.17$ \\
\hline 40, los & $\mathbf{9 9 . 1 , 0 . 9 8}$ & $97,0.97$ & $97,0.95$ & $44.6,0.35$ \\
\hline
\end{tabular}

We applied our proposed Parametric Link among Multinomial Mixtures (PLMM, Algorithm 1) clustering method on these simulated data using the basic sub-models defined in Sec. 3.3. Table 2 provides the results using the accuracy and ARI (Hubert and Arabie, 1985) measures. Moreover, it provides a comparative evaluation w.r.t. other state-of-the-art methods (see comparison in Table 1): (a) Temporal Multinomial Mixture (TMM) (Kim et al., 2015) with smoothness parameter $\alpha=1$; (b) Dynamic Topic Model (DTM) (Blei and Lafferty, 2006) with hyper-parameter $\alpha=0.01$ and (c) Adaptive evolutionary clustering method (AFFECT - we experimented AFFECT with hierarchical and spectral clustering also. However, k-means provided the best results) (Xu et al., 2014) with k-means and Euclidean distance as a measure of similarity. Besides the above settings, we set the maximum number of iteration to 500 for all of the above methods as the convergence criterion. We compute the average accuracy and ARI of time $t=2, \ldots, T$ (at $t=1$ there is no evolution). Results in Table 2 w.r.t. the accuracy and ARI show that:

- PLMM (proposed) provides the highest accuracy and ARI, even though it must be noted that the results from TMM (Kim et al., 2015) are often competitive. These results are not surprising as both PLMM and TMM methods are specialized to cluster samples which are drawn from the Multinomial distributions.

- DTM (Blei and Lafferty, 2006) provides better and competitive results for the los samples and higher dimensional data. This type of data is more likely from the text documents for which DTM was originally proposed. 
- $\operatorname{AFFECT}(\mathrm{Xu}$ et al., 2014) performs poorly compared to others for both types of sample. This is expected as the similarity measure used in AFFECT is appropriate for continuous data.

Table 3: Comparison among methods w.r.t. the computation time (in sec). Boldfaced indicate the lowest executation time.

\begin{tabular}{ccccc}
\hline & PLMM & TMM & DTM & AFFECT \\
\hline 10, hos & 0.39 & $\mathbf{0 . 0 6}$ & 9 & 0.66 \\
\hline $\mathbf{1 0}$, los & 24 & $\mathbf{0 . 1 2}$ & 1.8 & 0.19 \\
\hline $\mathbf{2 0}$, hos & 0.42 & $\mathbf{0 . 1}$ & 12 & 0.63 \\
\hline $\mathbf{2 0}$ los & 24 & $\mathbf{0 . 1}$ & 1.9 & 0.18 \\
\hline 40, hos & 0.55 & $\mathbf{0 . 1 4}$ & 10 & 0.62 \\
\hline 40, los & 29 & $\mathbf{0 . 1}$ & 2.1 & 0.18 \\
\hline
\end{tabular}

Next, we compare the methods based on the execution time requires for clustering. Table 3 provides the time (in sec), from which we see that TMM is the quickest method. For PLMM we observe that, hos samples clustering is significantly faster than the los samples. This is certainly a limitation of PLMM and we consider to improve it in our future work.

Next, we analyze the model estimation property based on its convergence to the true (generating) maximum value. To this aim, we compute the symmetric KL-Divergence (sKLD - we use the sKLD value because it has been used to compute the dissimilarities among the parameters of the Multinomial distributions Hasnat et al. (2015).) (see Sect. 3.6, Eq. 3.12) among the true generating parameters and the estimated model parameters. Our assumption for this evaluation is as follows: lower sKLD value indicates better estimation. In other word, a model approaches to the maxima when its estimated parameters are getting closer to the true generating parameters, i.e., the sKLD measure is closer to 0 . Table 4 presents the sKLD values of the PLMM and TMM methods. Here we consider only two methods because it was possible to obtain the MM model parameters from them and hence possible to compute the sKLD values. We have the following observations:

- PLMM provides better estimation (sKLD is lower) compared to the TMM. This observation supports the clustering accuracy and ARI measures of Table 2.

- Estimated parameters for the los samples are better (lower sKLD values) compared to the hos samples.

- Estimation quality decreases (higher sKLD values) with the increase of dimension. This is true for the hos samples with the PLMM method and both types of samples with the TMM method.

Table 4: Comparison among PLMM and TMM (Kim et al., 2015) based on the sKLD value among the true generating parameters and the estimated model parameters. Boldfaced indicate the better estimation based on sKLD values.

\begin{tabular}{ccc}
\hline & PLMM & TMM \\
\hline $\mathbf{1 0}$, hos & $\mathbf{3 . 4}$ & 3.6 \\
\hline $\mathbf{1 0}$, los & $\mathbf{0 . 3 3}$ & 0.5 \\
\hline $\mathbf{2 0}$, hos & $\mathbf{6 . 2}$ & 6.5 \\
\hline $\mathbf{2 0}$, los & $\mathbf{0 . 1 7}$ & 1.21 \\
\hline $\mathbf{4 0 ,}$ hos & $\mathbf{7 . 6}$ & 7.9 \\
\hline $\mathbf{4 0 ,}$ los & $\mathbf{0 . 1 9}$ & 1.7 \\
\hline
\end{tabular}

With the synthetic samples, we perform an additional analysis related to the required number of iterations (one of the convergence criteria). We observe that both PLMM and TMM converge before the specified maximum number of iterations, set to 500 (it was not possible to obtain the number of iterations required by the DTM and AFFECT methods from their available programs). Moreover, for the hos samples these methods converge faster than the los samples. We did not notice any dependency among the number of 
iterations and the dimension of the samples. On average, for the hos samples PLMM requires 10 iterations and TMM requires 50 iterations. For the los samples, PLMM requires 80 iterations and TMM requires 115 iterations.

Next, we analyze the evolution of the clusters in terms of the selected sub-models. Table 5 provides the rate of different selected models. We see that, for the hos data samples the model M4 $\left(1 / \gamma_{k, d}\right)$ is mostly selected. On the other hand, for the los data samples, different models M1: (1/0), M4: $\left(1 / \gamma_{k, d}\right)$ and M3: $\left(\delta_{k, d} / 0\right)$ are selected at certain rate. This observation confirms that PLMM successfully recovers the cluster evolutions with different models which were used to generate the simulated data. Interestingly, we observe that the model M2 $\left(0 / \gamma_{k, d}\right)$ is not selected. Based on the selected model, we can provide further interpretation using $\delta_{k, d}$ and $\gamma_{k, d}$, see Sec. 3.3.

Table 5: Percentage of the selected models for the interpretation of evaluation. hos: higher order (categorical count) samples and $l o s$ : lower order samples. Boldfaced indicate the highest rate.

\begin{tabular}{lcccc}
\hline & M1: $(1 / 0)$ & M4: $\left(1 / \gamma_{k, d}\right)$ & M3: $\left(\delta_{k, d} / 0\right)$ & M2: $\left(0 / \gamma_{k, d}\right)$ \\
\hline $\mathbf{1 0}, \boldsymbol{h o s}$ & $0 \%$ & $\mathbf{9 4} \%$ & $6 \%$ & $0 \%$ \\
\hline $\mathbf{1 0}, \boldsymbol{l o s}$ & $5 \%$ & $\mathbf{5 3} \%$ & $42 \%$ & $0 \%$ \\
\hline $\mathbf{2 0}, \boldsymbol{h o s}$ & $0 \%$ & $\mathbf{9 3} \%$ & $7 \%$ & $0 \%$ \\
\hline $\mathbf{2 0}, \boldsymbol{l o s}$ & $7 \%$ & $\mathbf{5 2} \%$ & $41 \%$ & $0 \%$ \\
\hline $\mathbf{4 0}, \boldsymbol{h o s}$ & $0 \%$ & $\mathbf{9 6} \%$ & $4 \%$ & $0 \%$ \\
\hline $\mathbf{4 0}, \boldsymbol{l o s}$ & $2 \%$ & $43 \%$ & $\mathbf{5 5 \%}$ & $0 \%$ \\
\hline
\end{tabular}

Finally, we conduct experiments with the varying number of clusters $K$ at different time epochs. For this experiment, we use the same MM parameters which were used to generate the hos data samples. To ensure different $K$ at different epoch, we randomly select a pair of time epochs and remove a cluster from one of them. Then, we generate $N^{t}=N^{t+1}=1000$ synthetic data samples from them using the same procedure mentioned before. Applying the extension of PLMM method (Section 3.6) on these data provides the following results (ARI): 0.967 for $d=10,0.988$ for $d=20$ and 0.986 for $d=40$. These results confirm that our proposed extension can cluster the synthetic data with varying $K$ and provides reasonable accuracy.

\subsection{Real data analysis: Opinion mining from twitter data}

In order to challenge the applicability of the proposed method on real world data we focus on a relevant dataset which: (a) consists of discrete/categorical data and (b) can be divided into multiple meaningful timestamps. To this aim, we collected data from the political opinion dataset of the ImagiWeb (http://mediamining.univ-lyon2.fr/velcin/imagiweb/dataset.html) (IW-POD) project Velcin et al. (2014). The motivation for choosing these data is that it consists of relatively lower number of features. Therefore, an evolution can be interpreted within a relatively easier and meaningful context.

IW-POD consists of manually annotated tweets, from May 2012 to January 2013, related to two French politicians: Francois Hollande (FH) and Nicolas Sarkozy (NS). First, these tweets are annotated into 11 different aspects, such as Attribute (Att), Person (Per), Entity (Ent), Skills (Skl), Political line (Pol), Balance (Bal), Injunction (Inj), Project (Pro), Ethic (Eth), Communication (Com) and No aspect detected $(\mathrm{N} / \mathrm{A})$. Afterward, each aspect is annotated with 6 opinion polarities, such as very negative $(-2)$, negative (1 ), no polarity (0), Null, positive $(+1)$ and very positive $(+2)$. For example, the tweet - Sarko is more rational (orig: Sarko est plus rationnel) is annotated with the aspect called Person and polarity +1 . It is about NS and indicates that the user provides positive opinion with an emphasis on the personal attribute. Another example, the tweet - Nicolas Sarkozy, the worst president of the Fifth Republic (Orig: Nicolas Sarkozy, le plus mauvais prsident de la Vme Rpublique) is annotated with the aspect called Skill and polarity -1 . It is a negative opinion about NS and indicates that the user emphasizes on the skill of NS.

In order to use these tweets for clustering, they are regrouped within the specified time epochs. Moreover, similar polarities are merged, e.g., two positives $(+1$ and +2$)$ are merged into one as only positive $(+)$. Therefore, each aspect consits of four polarities, such as positive $(+)$, negative (-), zero (0) and undefined/null $(\emptyset)$. As a consequence, finally each regrouped tweet is a $44(11 \times 4)$ dimensional vector of discrete data. In 
our experiment, we group the opinions from the IW-POD into three time epochs: $t 1, t 2$ and $t 3$. Table 6 provides the details of the temporal data. Since the true number of clusters is unknown, we run clustering for different numbers of clusters ranging from 3 to 9 .

Table 6: Details of the IW-POD dataset which is divided into three time periods. Each observation consists of a 44 dimensional discrete valued vector that encodes information about 11 different aspects each having 4 polarities.

\begin{tabular}{llcll}
\hline $\begin{array}{c}\text { Time } \\
\text { stamp }\end{array}$ & $\begin{array}{l}\text { Time } \\
\text { period }\end{array}$ & Significance & $\begin{array}{c}\text { Num. opinions } \\
\text { N. Sarkozy }\end{array}$ & $\begin{array}{c}\text { Num. opinions } \\
\text { F. Hollande }\end{array}$ \\
\hline t1 & $03 / 12-06 / 12$ & $\begin{array}{c}\text { Before and } \\
\text { After Election }\end{array}$ & 1018 & 1168 \\
\hline t2 & $07 / 12-10 / 12$ & After Election & 1067 & 1079 \\
\hline t3 & $11 / 12-01 / 13$ & After Election & 1079 & 708 \\
\hline
\end{tabular}

\subsubsection{Comparison among different methods}

We consider three different methods, Dynamic Topic Model (DTM) (Blei and Lafferty, 2006), Temporal Multinomial Mixture (TMM) (Kim et al., 2015) and Parametric Link among Multinomial Mixtures (PLMM), for a comparative evaluation on the IW-POD dataset. These methods are selected based on their specialty to cluster discrete evolutionary/temporal data. We set 100 maximum number of iterations as the convergence criterion for all methods (these values are set empirically based on the observations from our experiments on the convergence issue). Besides, we set the threshold log-likelihood difference values as 0.0001 for PLMM and TMM. The smoothness parameter $\alpha$ of TMM was set to 1 . The DTM hyper-parameter $\alpha$ was set to 0.01. For the PLMM method, we consider the sub-models mentioned in Sec. 3.3.

IW-POD dataset does not provide ground truth cluster labels. Therefore, we were unable to evaluate clustering results with the known-labels based metric, such as the accuracy and $A R I$. In this context, we evaluate the methods using a well known likelihood related measure called perplexity on a held-out test set (Murphy, 2012; Blei et al., 2003). Perplexity is a quantity originally used in the field of language modeling (Murphy, 2012). It measures how well a model has captured the underlying distribution of language. In clustering context, perplexity is defined as the reciprocal geometric mean of the per feature (word) loglikelihood of a test set. It is computed using the model parameters learned with a training set. The lower perplexity value indicates that the estimated (trained) model performs better to fit the test data. Perplexity can be formally defined as (Blei et al., 2003):

$$
\operatorname{perplexity}\left(X^{\text {test }}\right)=\exp \left(-\frac{L\left(\Theta^{\text {train }}\right)}{\sum_{i=1}^{N^{\text {test }}} V_{i}}\right)
$$

where, $V_{i}$ is the total number of feature counts (words for document) in observation $i, L\left(\Theta^{\text {train }}\right)$ denotes the log-likelihood of the test data set computed using the trained model parameters $\Theta^{\text {train }}$ and Eq. (3.6).

In our experiments, for each time epoch $t$, we compute perplexity from 5 folds of training-test data division and then take the average as the final measure. For each fold, we used $80 \%$ data for training and the remaining $20 \%$ data to compute perplexity. Fig. 4.1 illustrates the perplexity values computed from the data of two entities (row-1: Sarkozy and row-2: Hollande) and two time epochs (column-1: epoch $t 2$ and column-2: epoch $t 3$ ). Time epoch $t 1$ is not considered because it does not reflect the link relationship and temporal aspect of data clustering.

Results in Fig. 4.1 show that, PLMM provides the best perplexity compared to DTM and TMM. This means that, compared to other methods, PLMM provides better fitting of the underlying Multinomial distribution to the test data. The next best (3 out of 4 ) method is the DTM followed by the TMM. Indeed, the results from TMM are intuitive as the fitted models are highly influenced by the other cluster components (Multinomial distributions) from the previous and next time epochs. In contrary, PLMM only consider the link from one cluster in the previous time epoch and fit the data accordingly.

Fig. 4.2 provides a visual illustration of the clustering results obtained from the above three methods. It is obtained by using the Multidimensional scaling (Kruskal and Wish, 1978) technique, where the distance 


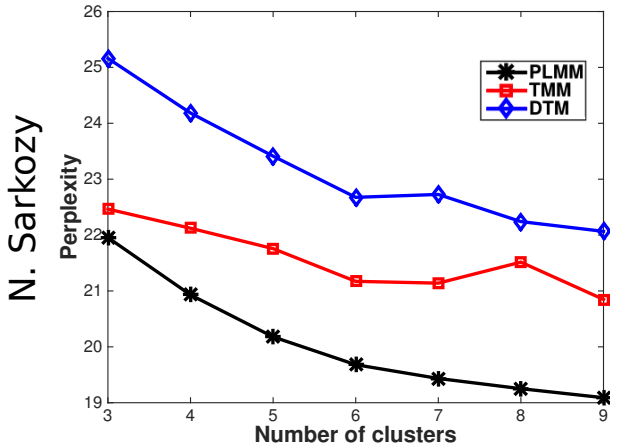

(a)

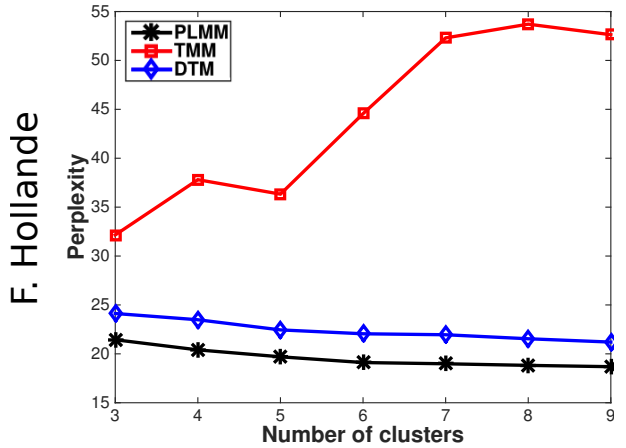

(c)

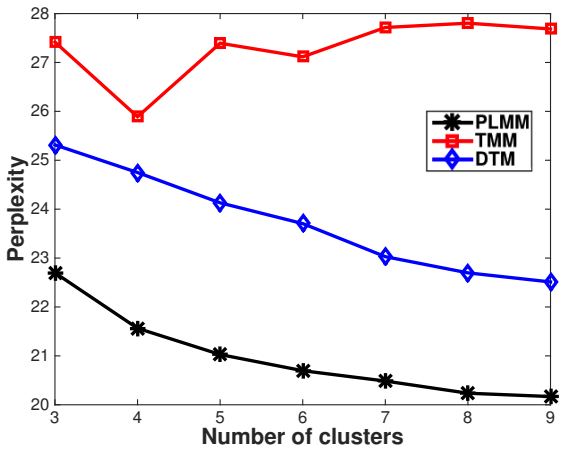

(b)

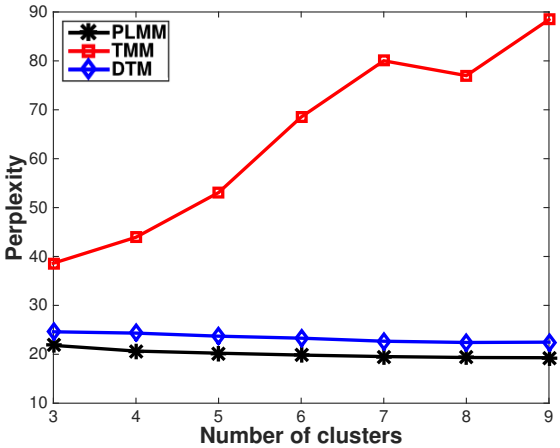

(d)

t2

Figure 4.1: Comparison of different methods w.r.t. the perplexity values (lower is better) computed from the IW-POD data of two entities (row-1: Sarkozy and row-2: Hollande) and two time epochs (column-1: epoch t2 and column-2: epoch t3). Methods: Dynamic Topic Model (DTM) (Blei and Lafferty, 2006), Temporal Multinomial Mixture (TMM) (Kim et al., 2015) and our proposed Parametric Link among Multinomial Mixtures (PLMM) method.

matrix among the observations is computed by first converting the count vectors into probabilities and then using the sKLD (Eq. 3.12) as a measure of distance. The clustering results are obtained with $K=3$, time epoch $t 2$ and the observations associated with the entity NS. From visual comparison among the plots in Fig. 4.2, we see that PLMM provides better separation than TMM and DTM. Indeed, this observation agrees with the numerical results obtained from the perplexity values in Fig. 4.1(a) for $K=3$.

Next, we apply the extension of PLMM method (Section 3.6) with this dataset and observe the perplexity for time epochs $t 2$ and $t 3$. For the entity NS, we obtain average perplexity values as: $t 2: 26.56$ and $t 3: 25.06$ where average $K^{t 2}$ is 3 and average $K^{t 3}$ is 5 . For the entity FH, we obtain average perplexity values as: $t 2: 13.08$ and $t 3: 5.17$ where average $K^{t 2}$ is 4 and average $K^{t 3}$ is 5 . Compared to the results in Fig. 4.1 we see that, perplexity values increases (performance decreases) for entity NS and decreases (performance improves) for FH. Based on these observations, we can say that the extension of PLMM provides a good compromise in performance and works well for varying $K$ at different epochs. We do not compare these results with the TMM and DTM methods as they work with fixed $K$ for all time epochs.

Finally, let us focus on the interpretations of cluster evolutions in the IW-POD dataset. Table 7 provides the selection rate of different models at different time epochs (see Table 6 for details of time division). Listed rates provide us very interesting observations:

- The opinions about NS were evolving almost similar way during and after the election period. These evolutions can be interpreted through the belief on aspects using models M3:( $\left.\delta_{k, d} / 0\right)(93 \%)$ and M4:(1/ $\left.\gamma_{k, d}\right)(7 \%)$. This indicates that during $t 1-t 2-t 3$ opinions about NS were changing slowly.

- Model M2: $\left(0 / \gamma_{k, d}\right)$ is selected for all clusters of opinions about FH during t1-t2. This means that the 


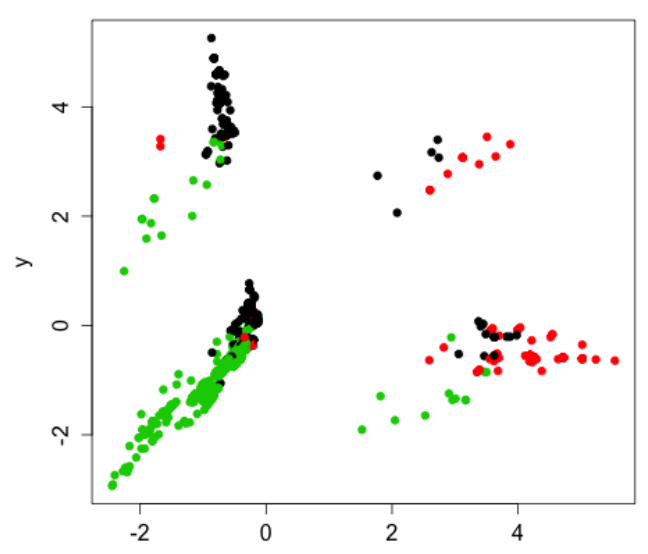

(a) PLMM

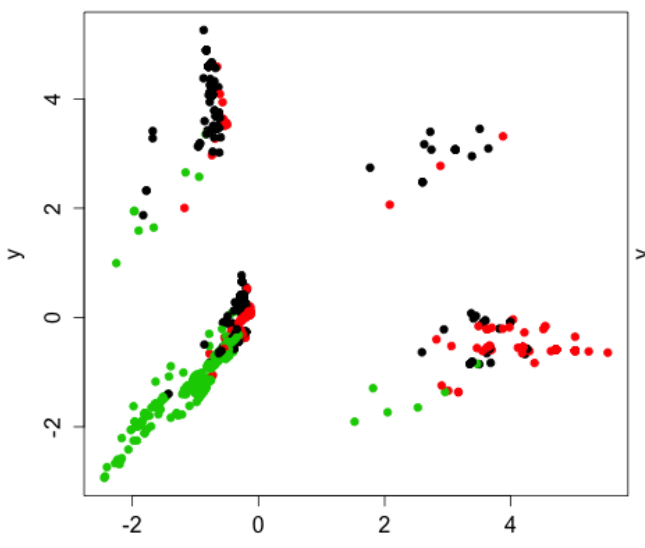

(b) $\stackrel{x}{\text { TMM }}$

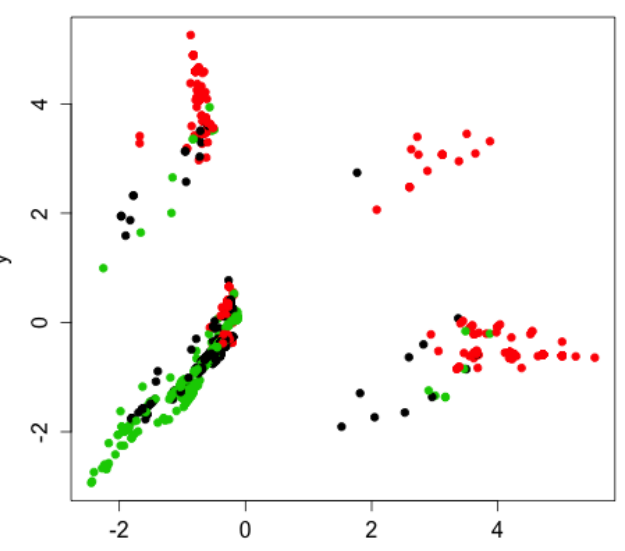

(c) $\stackrel{x}{\text { DTM }}$

Figure 4.2: Illustration of clustering results visualized with Multidimensional scaling (Kruskal and Wish, 1978). Methods: (a) proposed Parametric Link among Multinomial Mixtures (PLMM); (b) Temporal Multinomial Mixture (TMM) (Kim et al., 2015) and (c) Dynamic Topic Model (DTM) (Blei and Lafferty, 2006).

opinions change significantly between $t 1$ and $t 2$ period. From $t 2$ to $t 3$ (both after election period), opinions were evolving, which can be interpreted through the belief on the features with the models M4: $\left(1 / \gamma_{k, d}\right)(62 \%)$ and M3: $\left(\delta_{k, d} / 0\right)(38 \%)$.

\subsubsection{Cluster analysis, visualization and interpretation}

In this section, we analyze the clustering results only from the PLMM method. In order to visualize the contents, we construct a histogram representation. It is constructed by counting the polarities (in vertical direction) w.r.t. each attribute (in horizontal direction). The color of the bars resembles the color of polarities. Fig. 4.3 and 4.4 illustrate examples of the clusters at different time epochs for the entities NS and FH respectively. These results are obtained by clustering data with $K=3$. From both figures we observe that, at each time epoch the clusters have different histogram representations. Moreover, during different time epochs each cluster undergoes certain changes in different attributes and polarities. This demonstrates that PLMM method is able to provide sufficient inter-cluster variations (at each time) while respecting the temporal dynamics (during different time epochs). 
Table 7: Selection rate of different models (Sec. 3.3) for the IW-POD dataset at different time epochs (see Table 6 for details of time division)

\begin{tabular}{|c|c|c|c|c|}
\hline & M1: $(1 / 0)$ & M4: $\left(1 / \gamma_{k, d}\right)$ & M3: $\left(\delta_{k, d} / 0\right)$ & M2: $\left(0 / \gamma_{k, d}\right)$ \\
\hline$N S(t 1-t 2)$ & $0 \%$ & $0 \%$ & $100 \%$ & $0 \%$ \\
\hline$N S(t 2-t 3)$ & $0 \%$ & $13 \%$ & $87 \%$ & $0 \%$ \\
\hline$F H(t 1-t 2)$ & $0 \%$ & $0 \%$ & $0 \%$ & $100 \%$ \\
\hline$F H(t 1-t 2)$ & $0 \%$ & $62 \%$ & $38 \%$ & $0 \%$ \\
\hline
\end{tabular}

An alternative and compact representation (w.r.t. the MM model parameters) of the clusters for NS is illustrated in Fig. 4.5(a) and 4.5(b). Similar to the examples of Fig. 4.3, this alternative representation demonstrates that, at a certain time epoch, different clusters emphasize on different aspects/polarities of an entity. Besides, the temporal changes of the clusters can be identified subsequently during different epochs by observing the increase/decrease of the probabilities. However, from the user's perspective, this representation may not be convenient to understand. Therefore, we use histograms for further analysis and use this compact representation for a different purpose.

Now, let us explain the semantics obtained from these clustering results. For brevity, here we denote a cluster as cl.. From Fig. 4.3 (clusters for NS) we see that, while $c l .1$ and 3 emphasize on the negative (-) and positive (+) polarities respectively, $\mathrm{cl}$. 2 emphasizes on a particular attribute. Naively we can say that, there are three groups of peoples: (a) the first group ( $c l$. 1) provides negative opinions from various aspects, thus tends to hold a negative image about the entity; (b) the second group (cl. 2) particularly emphasizes on Ethic of the entity and mostly provide negative opinions and (c) the third group (cl. 3) can be seen as a contrary to the first group ( $c l .1)$ as it tends to hold a positive image about the entity. Table 8 provides three examples of the tweets for time $t 1$ and for each cluster about NS. We can realize that these tweets reflect the opinions which truly correspond to the groups obtained by the clustering method.

From temporal viewpoint, we observe several changes w.r.t. different aspects. In order to analyze the changes using histograms, we observe the height of histogram bar for each aspect. This height indicates the number of tweets/opinions corresponding to the related aspect. Let us consider an example of the aspect Communication, which plays a certain role on clustering. We observe that: (a) for $\mathrm{cl}$. 1 , the total number of tweets related to the aspect Communication remains same during time $t 1$ and $t 2$ and reduces during $t 2$ and $t 3$; (b) for $c l$. 2, the total number of tweets related to this aspect reduces continuously and (c) for $c l$. 3, the total number of tweets related to this aspect reduces from $t 1$ to $t 2$ and remains same during $t 2$ to $t 3$. Moreover, a closer look on $\mathrm{cl}$. 3 from $t 2$ to $t 3$ reveals an increase of positive opinions about the communication skill of the entity. Another example is the aspect called Attribute, whose height reduces continuously with time for both $c l .1$ and 3. Similarly, from an analysis of the height of histogram bars in Fig. 4.4 (clusters for FH) we see that, the aspects called Entity, Ethic, Political line, Skills and Communication play certain role to describe the image of FH. For example, the tweet - Holland would remove the word "race" in the Constitution (orig: Hollande supprimerait le mot "race" dans la Constitution) from time $t 1$ and $c l .3$ is annotated with the aspect called political line and polarity +1 . Another tweet - Holland and Netanyahu evoke the struggle against anti-Semitism (orig: Hollande et Netanyahou voquent la lutte contre l'antismitisme) has the same annotation which is from the same cluster but from time $t 3$. These two examples reveal the importance of the aspect political line for keeping the similar opinions into the same group at different time. The above observations clearly indicate that, for different groups of people different aspects has certain importance at different time. Therefore, an analyst can retrieve the most prominent aspects from people's opinion about an entity at a particular time or within a certain range of time periods.

Besides the above interpretation of the clustering results, an analyst can obtain more information from the PLMM clustering results via the link parameters $\left(\delta_{k, d}\right.$ or $\left.\gamma_{k, d}\right)$. After analyzing the links among the MM model parameters, we notice that they are able to provide a compact explanation about the temporal changes during two time epochs. Fig. 4.5 illustrates an example for entity $N S$ from time $t 1$ to $t 2$ with 3 clusters, see column 1 and 2 of Fig. 4.3 for corresponding histograms. Fig. 4.5(a) and Fig. 4.5(b) illustrates the MM parameters (probabilities of the aspect-polarity features) and Fig. 4.5(c) provides a compact representation 


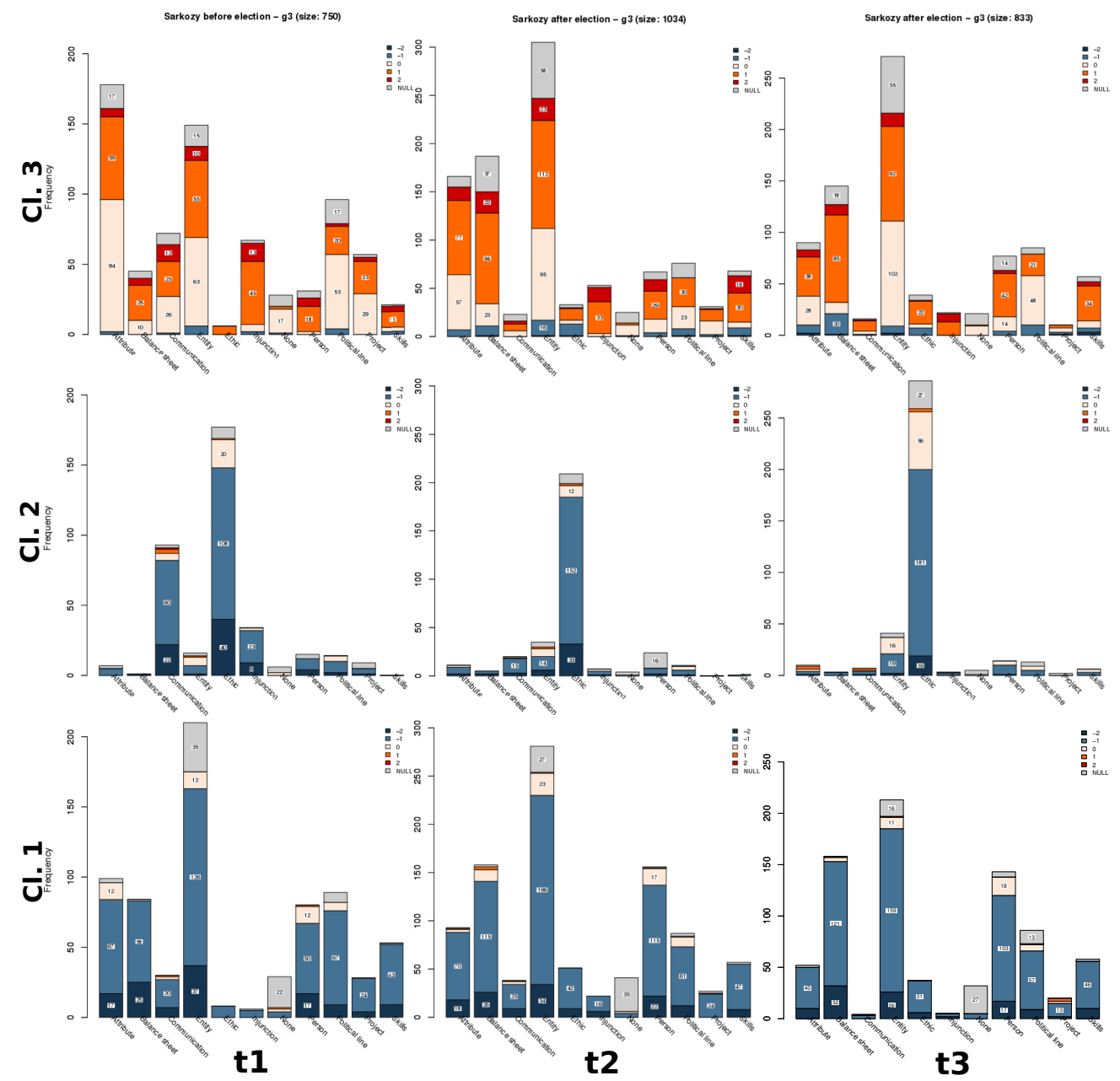

Figure 4.3: Illustration of the clustering results from the PLMM methods for NS. Results obtained using $K=3$ for three time epochs $t 1, t 2$ and $t 3$. Each cluster is represented as a histogram constructed from the polarities of different aspects. The aspects are ordered from left to right as: (1) Attribute; (2) Balance sheet; (3) Communication; (4) Entity; (5) Ethic; (6) Injunction; (7) None; (8) Person; (9) Political line; (10) Project and (11) Skills. The polarities are colored and ordered from bottom to top as: -2 (dark blue), -1 (blue), 0 (light orange), 1 (orange), 2 (red) and NULL (grey). Each column represents clusters from a particular epoch. Each row represents a particular cluster in different epochs. 

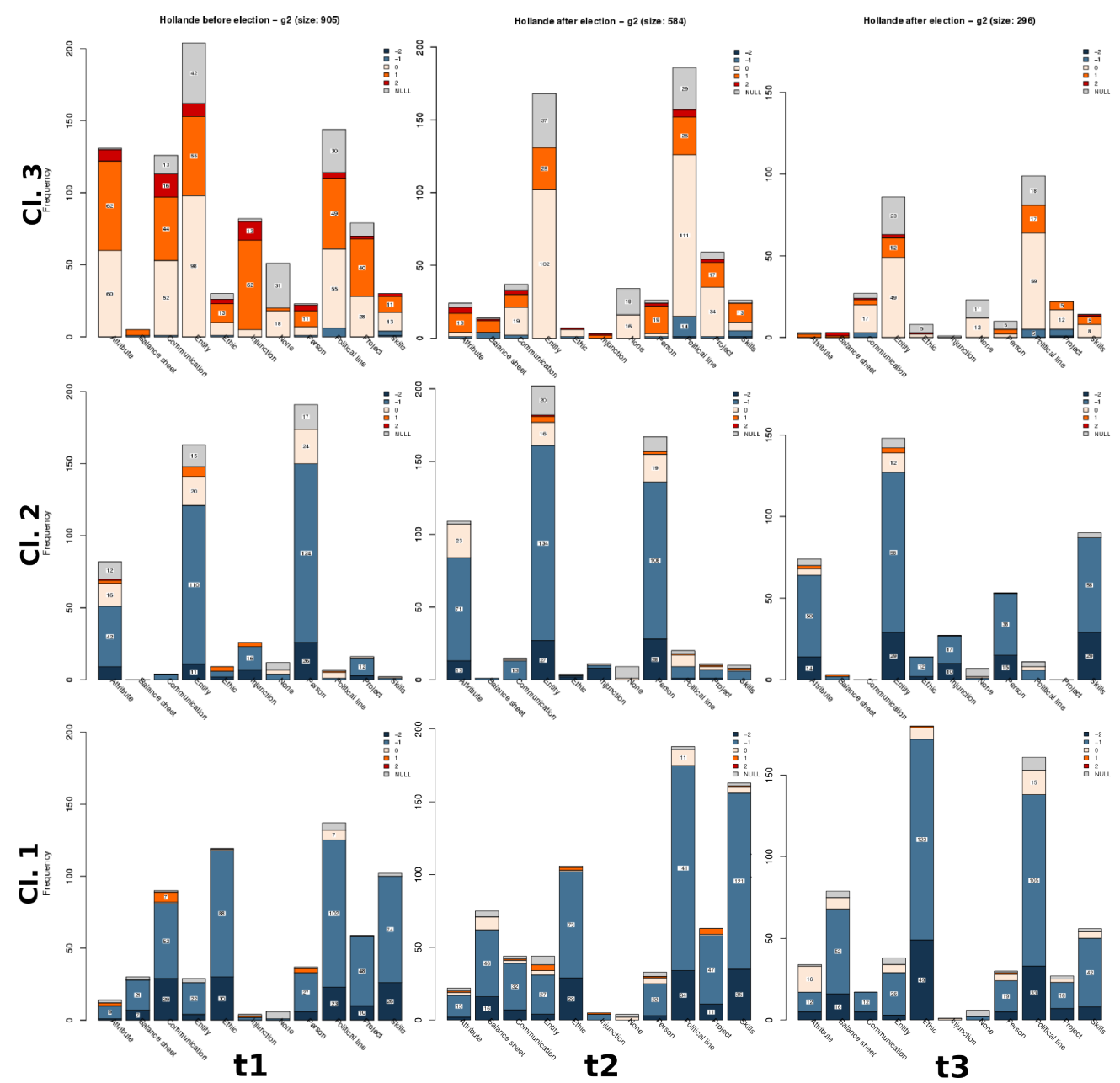

Figure 4.4: Illustration of the clustering results from the PLMM methods for FH. Results obtained using $K=3$ for three time epochs $t 1, t 2$ and $t 3$. Each cluster is represented as a histogram constructed from the polarities of different aspects. The aspects are ordered from left to right as: (1) Attribute; (2) Balance sheet; (3) Communication; (4) Entity; (5) Ethic; (6) Injunction; (7) None; (8) Person; (9) Political line; (10) Project and (11) Skills. The polarities are colored and ordered from bottom to top as: -2 (dark blue), -1 (blue), 0 (light orange), 1 (orange), 2 (red) and NULL (grey). Each column represents clusters from a particular epoch. Each row represents a particular cluster in different epochs. 
Table 8: Real twitter data examples of the 3 clusters at time $t 1$ for entity NS. See Fig. 4.3 column 1 for the associated histograms.

Cluster 1 (Generally Negative)

Orig: Il veut des rfrendums car... y a pas de pilote dans l'avion,

dit-il: quel aveu! \#Sarkozy\#projet

Ex. 1 Trans: He wants referendum because there is no pilot in the plane he says: what a confession! \#Sarkozy\#project

Ex. 2 Orig: Je ne voterais pas \#Sarkozy !" " Je ne voterais pas \#Sarkozy!

Trans: I won't vote for \#Sarkozy !" " I won't vote for \#Sarkozy

Ex. 3 Orig:Nicolas Sarkozy, le plus mauvais prsident de la Vme Rpublique

Trans: Nicolas Sarkozy, the worst president of the Fifth Republic

$$
\text { Cluster } 2 \text { (Negative, specially "Ethic") }
$$

Orig: Jamais un prsident n'a t cern par tant d'affaires! demain ds

Ex. 1 @lematinch \#Bettencourt \#Sarkozy

Trans: Never before a president was surrounded by so many cases! tomorrow in @lematinch \#Bettencourt \#Sarkozy

Orig: Une liste de condamns de l'\#UMP qui pourrait tre bientt complte par les noms de \#Sarkozy, \#Cop, \#Woerth

Ex. 2 Trans: A list of convicted people of \#UMP soon completed by names such as \#Sarkozy, \#Cop, \#Woerth (the Bettencourt case is a famous case in which Sarkozy was involved)

Orig: Sarkozy-Kadhafi: la preuve du financement. Et l'urgence d'une enqute officielle \#affairedetat

Ex. 3 Trans: Sarkozy-Kadhafi: the proof of funding. And the urge of an official enquiry \#stateaffair (Kadhafi is another case in which Sarkozy was involved in some way) Cluster 3 (Generally Positive)

Orig: N Sarkosy mots cl..challenge, dfi, action, travail, russite, formation, effort, individualisation ..France Forte. Europe Forte \#NS2012

Ex. 1 Trans: N Sarkozy keywords..challenge, dfi, action, work, success, training, effort, individualization ...Strong France. Strong Europe \#NS2012

Ex. 2 Orig: merci N.Sarkozy pour tout tu restera pour toujour mon Hero merci. merci Trans: Thank you N.Sarkozy for all you will stay my hero forever thanks. thanks

Orig: Sarko est plus rationnel.

Trans: Sarko is more rational. 
about the cluster evolutions using the values of $\delta_{k, d}$. To better understand this representation in Fig. 4.5(c), we transform the link values as 0 (no change), $-1\left(\delta_{k, d}<0.9\right.$, belief increases) and $+1\left(\delta_{k, d}>1.1\right.$, belief decreases). In the context of the examples from the IW-POD, we can explain the belief as: probability of a feature at time $t+1$ is increased from its probability at time $t$. Therefore, the belief indicates the relative significance of a particular feature w.r.t. time. An increase in the belief means that users tend to be more attracted by it. Following this, if a feature probability is nearly same at two different times then belief remains unchanged. In Fig. 4.5, we highlight the effect of a particular aspect, called Communication $(\mathrm{Com})$, and observe its contribution for cluster evolution. From Fig. 4.5 (a) and (b) we see that, from time $t 1$ to $t 2$ the probabilities are decreased mostly for $c l$. 2 and 3. This means that, either the users from these clusters loose interest to discuss about Com and focus on other aspects, or those users disappeared at time t2. Similar to Com, we can observe other aspects such as Eth (cl. 1 and cl. 3) and Ent (cl. 2 and cl. 3) which causes cluster evolution in this example of Fig. 4.5.

Let us analyze examples from real twitter data and observe them w.r.t. Fig. 4.5. If we look at $c l$. 3 at $t 1$ (before election), the most likely features are often positive and it is clear that it gathers people in favor of NS. The prominent aspects are Att (positive and neutral), Ent (positive) and Inj (positive), such as in the tweet - 40 people@youngpop44will be present at the great gathering in Place \#Concorde for supporting @NicolasSarkozy! \#StrongFrance \#NS2012". This cluster slightly changes later at t2 (just after election) towards Att (positive), Ent (positive) and Bal (positive). The shift from Inj to Bal is clearly visible on Fig. 4.5(c), third row: black color for Inj means a decrease of attention whereas white color for Bal means there are relatively more comments on the balance sheet of NS. Hence, the following message shows some nostalgia felt by many militants: Whatever the opinion of FH, NS has been a great president. FH can deconstruct all the reforms, we will never forget!. To sum up, the $\delta$ parameter helps us to focus on what are the main changes, even though the observations could have been drawn among the other aspects. Following the same reasoning, all polarities targeting the aspect Com are black, which proves that the performances of the politician in the media (e.g., TV, newspapers) are less important once the election is over.

Observations from numerous experiments reveal that, besides performing evolutionary clustering on the temporal data, PLMM also provide reasonable interpretation for the evolutions, thanks to the link parameters. Indeed, this clearly distinguishes PLMM from the rest of the state-of-the-art methods. Moreover, we notice that the interpretability of PLMM (using Eq. 3.9, 3.10 and 3.11) can be separated out and externally plugged-in with the results from any other discrete data clustering methods.

\section{Conclusion and Future Perspectives}

Over the years, a large number of temporal data analysis methods have been proposed in several domains. In this paper, we only focused on the particular clustering methods which have been used for discrete data clustering and which are based on the assumption of the Multinomial distribution.

We proposed an unsupervised method (i.e., no training from labeled data) for analyzing the temporal data. The core element of our proposal is the formulation of parametric links among the Multinomial distributions. Computations of these links naturally cluster the evolutionary/temporal data. Furthermore, these links can provide interpretation for cluster evolution and also detect clusters evolution in certain cases. For experimental validation, we extensively used synthetic dataset and evaluated using the Clustering Accuracy and Adjusted Rand Index. As a practical application, we applied it on a dataset of political opinions and evaluated using the Perplexity measure. Results show that the proposed method, called PLMM, is better than the state-of-the-art. Moreover, it provides an additional advantage through the link parameters in order to interpret the changes in clusters at different time. We also provide an extension of the proposed method for dealing with varying number of clusters, which is not addressed by most of the recent methods.

Monitoring/tracking cluster evolution is an interesting issue which we do not explicitly and extensively manage in our proposed method, because it is not a primary objective in this paper. Yet, we can partially achieve this task by using certain information (parametric sub-models, see 3.3) which are naturally integrated with our proposed method. That means, our method can be used only as a detector of cluster evolution. At present, we consider the complete monitoring task as a future work. We believe that, several existing work can be added with our method to completely deal with this issue. For example, we can exploit MEC 


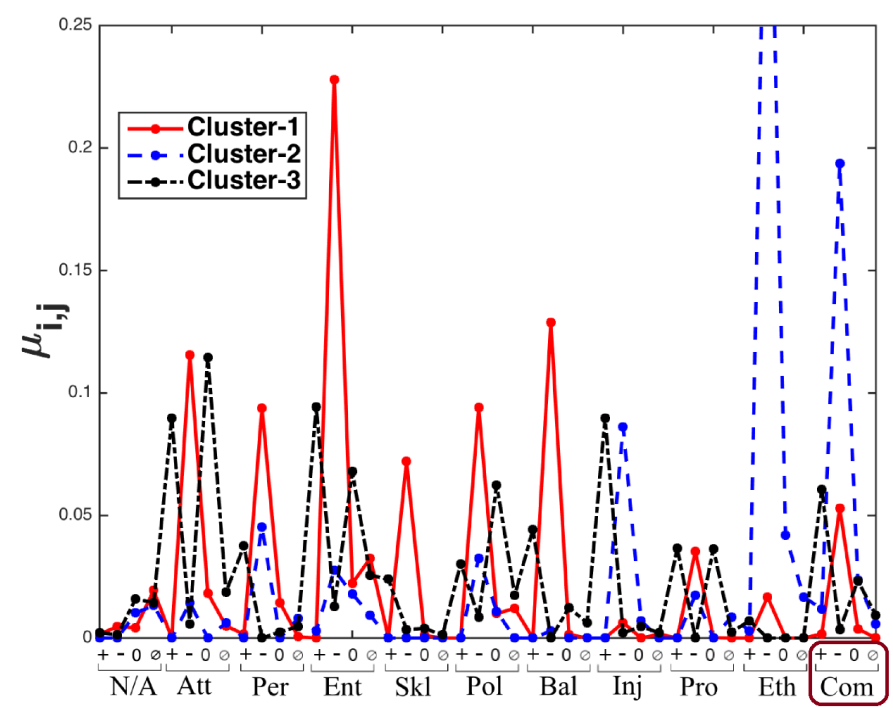

(a)

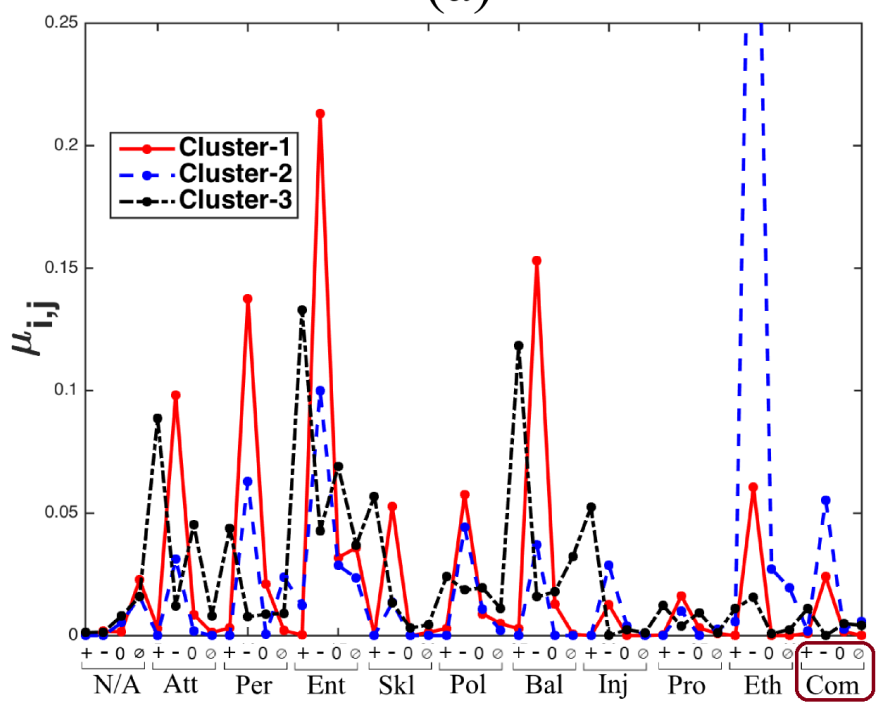

(b)

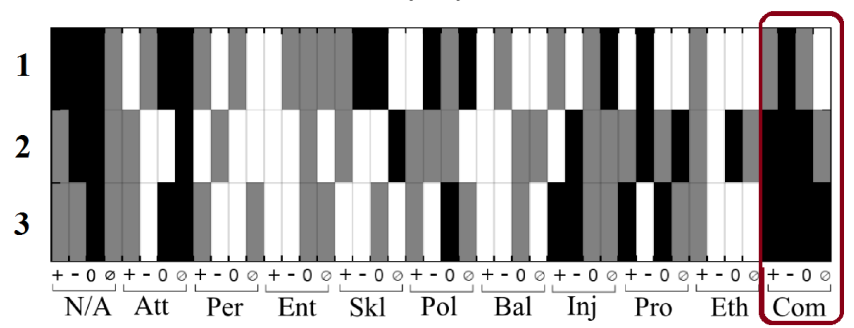

(c)

Figure 4.5: Example of evolution interpretation $\delta_{k, d}$ for NS during t1 to t2 with 3 clusters. (a) MM parameters $\mu_{k, j}^{t 1}$ at time t1 (b) MM parameters $\mu_{k, j}^{t 2}$ at time $t 2$ (c) Link parameters $\delta_{k, j}$ between time $t 1$ and $t 2$. In (c), for each cluster (row-wise), brighter/white color indicates the prior belief about features (aspect-polarity) increases, darker/black color indicates the prior belief about features decreases and grey color indicates the prior belief about features remains same. 
(Oliveira and Gama, 2010) which is a cluster evolution monitoring method for continuous data. Besides, we can use the label-based diachronic approach (Lamirel, 2012) by externally providing our clustering results as an input to it.

Computational complexity is a concern for the proposed method and can be considered as a limitation. From a decomposition of the computational time, we observe that most of the time is consumed by the optimization procedure (neldermead simplex method). In future, a better optimization method can be incorporated to address this issue. Moreover, the time can be further reduced by eliminating the parametric sub-models which are experimentally found as redundant.

Although we demonstrated the effectiveness of the proposed method only for the political opinion dataset, we believe that it will be equally effective for different datasets that consist of the form of categorical data.

\section{Acknowledgements}

This work is funded by the project ImagiWeb ANR-2012-CORD-002-01.

\section{References}

Agresti, A., 2002. Categorical data analysis, 2nd Edition. John Wiley \& Sons.

Allman, E., Matias, C., Rhodes, J., 2009. Identifiability of parameters in latent structure models with many observed variables. Annals of Statistics 37 (6A), 3099-3132.

Baudry, J.-P., Celeux, G., 2015. EM for mixtures-initialization requires special care. Statistics and Computing 25 (4), $713-726$.

Beninel, F., Biernacki, C., Bouveyron, C., Jacques, J., Lourme, A., 2012. Parametric link models for knowledge transfer in statistical learning. Knowledge Transfer: Practices, Types and Challenges. Nova Science Publishers.

Biernacki, C., Beninel, F., Bretagnolle, V., 2002. A generalized discriminant rule when training population and test population differ on their descriptive parameters. Biometrics 58 (2), 387-397.

Biernacki, C., Celeux, G., Govaert, G., 2000. Assessing a mixture model for clustering with the integrated completed likelihood. IEEE TPAMI $22(7), 719-725$.

Biernacki, C., Celeux, G., Govaert, G., 2003. Choosing starting values for the EM algorithm for getting the highest likelihood in multivariate gaussian mixture models. Computational Statistics \& Data Analysis 41 (3), 561-575.

Biernacki, C., Celeux, G., Govaert, G., Langrognet, F., 2006. Model-based cluster and discriminant analysis with the MIXMOD software. Computational Statistics \& Data Analysis 51 (2), 587-600.

Bishop, C. M., et al., 2006. Pattern recognition and machine learning. Vol. 4. springer New York.

Blei, D. M., Lafferty, J. D., 2006. Dynamic topic models. In: Proc. of the Int Conf on Machine Learning. ACM, pp. 113-120.

Blei, D. M., Ng, A. Y., Jordan, M. I., 2003. Latent Dirichlet allocation. Journal of Machine Learning Research 3, $993-1022$.

Chakrabarti, D., Kumar, R., Tomkins, A., 2006. Evolutionary clustering. In: Proceedings of the 12th ACM SIGKDD international conference on Knowledge discovery and data mining. ACM, pp. 554-560.

Chi, Y., Song, X., Zhou, D., Hino, K., Tseng, B. L., 2009. On evolutionary spectral clustering. ACM Trans. on Knowledge Discovery from Data $3(4), 17$.

Dubey, A., Hefny, A., Williamson, S., Xing, E. P., 2013. A nonparametric mixture model for topic modeling over time. In: SDM. SIAM, pp. 530-538.

Ferlez, J., Faloutsos, C., Leskovec, J., Mladenic, D., Grobelnik, M., 2008. Monitoring network evolution using MDL. In: IEEE Int. Conf. on Data Engineering. IEEE, pp. 1328-1330.

Figueiredo, M. A. T., Jain, A. K., 2002. Unsupervised learning of finite mixture models. IEEE TPAMI 24 (3), $381-396$.

Fraley, C., Raftery, A. E., 2002. Model-based clustering, discriminant analysis, and density estimation. Journal of the American Statistical Association 97 (458), 611-631.

Garcia, V., Nielsen, F., 2010. Simplification and hierarchical representations of mixtures of Exponential families. Signal Processing 90 (12), 3197-3212.

Hasnat, M. A., Alata, O., Trémeau, A., 2016. Model-based hierarchical clustering with Bregman Divergences and Fishers mixture model: application to depth image analysis. Statistics and Computing 26 (4), 861-880.

Hasnat, M. A., Velcin, J., Bonnevay, S., Jacques, J., 2015. Simultaneous clustering and model selection for Multinomial distribution: A comparative study. In: Advances in Intelligent Data Analysis XIV. Springer.

Hubert, L., Arabie, P., 1985. Comparing partitions. Journal of classification 2 (1), 193-218.

Jacques, J., Biernacki, C., 2010. Extension of model-based classification for binary data when training and test populations differ. Journal of Applied Statistics 37 (5), 749-766.

Kharratzadeh, M., Renard, B., Coates, M., 2015. Bayesian topic model approaches to online and time-dependent clustering. Digital Signal Processing.

Kim, Y.-M., Velcin, J., Bonnevay, S., Rizoiu, M.-A., 2015. Temporal Multinomial mixture for instance-oriented evolutionary clustering. In: Proc. of the European Conference on Information Retrieval. pp. 593-604.

Kruskal, J. B., Wish, M., 1978. Multidimensional scaling. Vol. 11. Sage.

Lamirel, J.-C., 2012. A new approach for automatizing the analysis of research topics dynamics: application to optoelectronics research. Scientometrics 93 (1), 151-166. 
McLachlan, G. J., Krishnan, T., 2008. The EM algorithm and extensions, 2nd Edition. Wiley series in probability and statistics. Wiley.

Meilă, M., Heckerman, D., 2001. An experimental comparison of model-based clustering methods. Machine Learning 42 (1-2), 9-29.

Melnykov, V., Maitra, R., 2010. Finite mixture models and model-based clustering. Statistics Surveys 4, 80-116.

Murphy, K. P., 2012. Machine learning: a probabilistic perspective. The MIT Press.

Nelder, J. A., Mead, R., 1965. A simplex method for function minimization. The computer journal 7 (4), $308-313$.

Oliveira, M. D., Gama, J., 2010. MEC-monitoring clusters' transitions. In: STAIRS. pp. $212-224$.

Salvador, S., Chan, P., 2004. Determining the number of clusters/segments in hierarchical clustering/segmentation algorithms. In: IEEE Conf. on Tools with Artificial Intelligence. pp. 576-584.

Schwarz, G., et al., 1978. Estimating the dimension of a model. The Annals of Statistics 6 (2), $461-464$.

Silvestre, C., Cardoso, M. G., Figueiredo, M. A., 2014. Identifying the number of clusters in discrete mixture models. arXiv preprint arXiv:1409.7419.

Spiliopoulou, M., Ntoutsi, I., Theodoridis, Y., Schult, R., 2006. MONIC: modeling and monitoring cluster transitions. In: Proc. of the ACM SIGKDD Int conf. on Knowledge discovery and data mining. ACM, pp. 706-711.

Velcin, J., Kim, Y., Brun, C., Dormagen, J., SanJuan, E., Khouas, L., Peradotto, A., Bonnevay, S., Roux, C., Boyadjian, J., et al., 2014. Investigating the image of entities in social media: Dataset design and first results. In: Proc. of Language Resources and Evaluation Conference (LREC).

Xu, K. S., Kliger, M., Hero Iii, A. O., 2014. Adaptive evolutionary clustering. Data Mining and Knowledge Discovery 28 (2), 304-336.

Xu, T., Zhang, Z., Yu, P. S., Long, B., 2008. Dirichlet process based evolutionary clustering. In: Data Mining, 2008. ICDM'08. Eighth IEEE International Conference on. IEEE, pp. 648-657.

Xu, T., Zhang, Z., Yu, P. S., Long, B., 2012. Generative models for evolutionary clustering. ACM Trans. on Knowledge Discovery from Data $6(2), 7$.

Ypma, J., 2014. Introduction to nloptr: an $\mathrm{r}$ interface to nlopt.

Zhong, S., Ghosh, J., 2005. Generative model-based document clustering: a comparative study. Knowledge and Information Systems 8 (3), 374-384. 